\title{
Molecular Interactions at Vapor-Liquid Interfaces: Binary Mixtures of Simple Fluids
}

\author{
Simon Stephan ${ }^{1, \text { a) }}$ and Hans Hasse ${ }^{1}$ \\ Laboratory of Engineering Thermodynamics (LTD), TU Kaiserslautern, \\ 67663 Kaiserslautern, Germany
}

(Dated: 24 January 2020)

Properties of vapor-liquid equilibria and planar interfaces of binary Lennard-Jones truncated and shifted (LJTS) mixtures were investigated with molecular dynamics (MD) simulations, density gradient theory (DGT), and conformal solution theory (CST) at constant liquid phase composition and temperature. The results elucidate the influence of the liquid phase interactions on the interfacial properties (surface tension, surface excess, interfacial thickness, and enrichment). The studied mixtures differ in the ratios of the dispersion energies of the two components $\varepsilon_{2} / \varepsilon_{1}$ and the binary interaction parameter $\xi$. By varying $\xi$ and $\varepsilon_{2} / \varepsilon_{1}$, a variety of types of phase behavior is covered by the study. The dependence of the interfacial properties on the variables $\xi$ and $\varepsilon_{2} / \varepsilon_{1}$ reveals regularities that can be explained by conformal solution theory of the liquid phase. It is thereby shown that the interfacial properties of the mixtures are dominated by the mean liquid phase interactions whereas the vapor phase has only a minor influence.

Keywords: interfacial properties, Molecular Dynamics, Density Gradient Theory, Conformal Solution Theory, Lennard-Jones truncated shifted, interfacial enrichment

a) Electronic mail: Simon.Stephan@mv.uni-kl.de 


\section{INTRODUCTION}

Properties of vapor-liquid interfaces play a crucial role in many chemical engineering applications, such as distillation and absorption. Experimental data on interfacial properties are in most cases limited to surface tension, from which sometimes data on the adsorption at the fluid interface is derived ${ }^{1,2}$. Hence, reliable and predictive models for such properties are an essential need.

On a molecular level, fluid interfaces are three-dimensional objects. In the interfacial region, which is generally only a few nanometers wide, the density changes smoothly from the value in one bulk phase to that in the other. This holds for the total density as well as for the component densities. Experimental methods for the investigation of such density profiles on a molecular level are presently not available. However, density profiles can be obtained by theoretical methods ${ }^{3,4}$, namely by molecular simulation based on classical force fields on the one side and density gradient theory (DGT) or density functional theory (DFT) combined with an equation of state (EOS) of the fluid on the other side. In the present work both molecular dynamics (MD) simulation and DGT are used to conduct a comprehensive study of binary Lennard-Jones truncated and shifted (LJTS) mixtures, regarding their vapor-liquid equilibrium (VLE) and interfacial properties. Based on that data set, it is shown by applying a conformal solution theory (CST) that the mean molecular interactions in the liquid phase dominate the interfacial properties of the studied systems.

While the profile of the total density in the interfacial region increases monotonously from the bulk vapor to the bulk liquid phase, this does not necessarily hold for the profiles of all individual component densities. A maximum of the component density in the interfacial region is observed in many cases for the low-boiling component and can reach values several times larger than the largest value of the two bulk densities of that component ${ }^{1,5}$. We refer to this phenomenon as enrichment. The enrichment of components at the interface of mixtures is of particular interest as it is suspected to affect the mass transfer through the interface $^{6-8}$. Interfacial enrichment has been found in many mixtures in studies ${ }^{1,5,6,8-41}$ that were carried out with molecular simulations, DGT, and DFT, but no systematic evaluation of the phenomenon of interfacial enrichment has been conducted so far to elucidate its dependency on the type of the mixture. However, it is known that a strong enrichment is

favored by a wide-boiling behavior of the mixture ${ }^{1,5,8,14,26,29,31,35,36,42}$. The relation between 
the relative adsorption and the enrichment and their relation to the molecular interactions is not yet fully understood ${ }^{1,5}$.

While the global phase behavior of Lennard-Jones mixtures or mixtures of other simple spherical molecules has been investigated numerous times in the literature ${ }^{43-47}$, no systematic study of the global behavior of interfacial properties is available.

We have therefore carried out such a study using both molecular dynamics simulation and DGT. The focus lies on the interfacial adsorption and enrichment and its dependency on the molecular interactions. The studied fluid is described by the Lennard-Jones truncated and shifted (LJTS) potential. The PeTS EOS ${ }^{5,48}$ (Perturbed Lennard-Jones truncated and shifted) is used to describe the LJTS fluid within DGT. The planar vapor-liquid interface of 90 binary LJTS mixtures was investigated at constant temperature $\left(T=0.77 \varepsilon k_{\mathrm{B}}^{-1}\right)$ and constant liquid phase composition $\left(x_{2}^{\prime}=0.05 \mathrm{~mol} \mathrm{~mol}^{-1}\right)$. The temperature is 0.7 of the critical temperature of the high-boiling component.

The 90 investigated mixtures were obtained by varying the dispersion energies of the two components $\varepsilon_{2} / \varepsilon_{1}$ and the binary interaction parameter $\xi$ in the modified Berthelot combination rule ${ }^{49}$. The size parameter of both components was the same and was not varied in this work. For the planar interfaces of these mixtures the surface tension, interfacial thickness, surface excess, and enrichment of the low-boiling component was determined, such that a large body of data for elucidating the influence of the different interactions on the interfacial properties became available.

All interfacial properties of different studied mixtures were found to have common regularities regarding their $\xi, \varepsilon_{2} / \varepsilon_{1}$-dependency. Using a CST, it is shown that these regularities can be explained in terms of the mean liquid phase interactions, i.e. the internal energy. This methodology is used to correlate the interfacial properties as functions of the internal energy of the liquid phase. CST has been applied to bulk properties ( $\operatorname{static}^{50-55}$ and transport ${ }^{56}$ ) but to the best of our knowledge not to interfacial properties.

By computing the Henry's law constants of the investigated LJTS mixtures, the results and the physical insight from the interfacial properties behavior are furthermore put into relation with the gas solubility of the low-boiling component in the high-boiling component. 


\section{MODELING AND SIMULATION}

\section{A. Binary Lennard-Jones Truncated Shifted Mixtures}

One of the cheapest intermolecular potentials in terms of the required computational effort which still gives a correct description of the behavior of simple fluids is the Lennard-Jones truncated and shifted (LJTS) potential ${ }^{57,58}$ with a truncation radius of $2.5 \sigma$. It is therefore often used as model fluid in studies of physical phenomena ${ }^{59-63}$ and for the development of simulation methods.

The LJTS potential $v_{\text {LJTS }}$ is

$$
\begin{array}{r}
v_{\mathrm{LJ}}(r)=4 \varepsilon\left[\left(\frac{\sigma}{r}\right)^{12}-\left(\frac{\sigma}{r}\right)^{6}\right] \\
v_{\mathrm{LJTS}}(r)= \begin{cases}v_{\mathrm{LJ}}(r)-v_{\mathrm{LJ}}\left(r_{\mathrm{c}}\right) & r \leq r_{\mathrm{c}} \\
0 & r>r_{\mathrm{c}},\end{cases}
\end{array}
$$

with $v_{\mathrm{LJ}}$ being the full Lennard-Jones potential ${ }^{64,65}, \varepsilon$ and $\sigma$ being the energy and size parameter, respectively. The distance between two particles is denoted by $r$. The truncation radius $r_{\mathrm{c}}$ of the potential is $2.5 \sigma$ throughout the present work. The abbreviation 'LJTS' refers to the fluid with this specific truncation radius here. Note, that the truncation of the potential influences the fluid's thermodynamic bulk ${ }^{66-69}$ and interfacial properties ${ }^{70-75}$.

90 binary LJTS mixtures were investigated in the present work. The high-boiling component is denoted as component 1 and the low-boiling component as 2 . The size parameter of both components is the same for all investigated mixtures, i.e. $\sigma_{1}=\sigma_{2}=\sigma$. Also the mass of both components is the same. Component 1 is considered as reference component here and is the same for all mixtures with $\varepsilon_{1}=1$ and $\sigma_{1}=1$. The low-boiling character of component 2 is obtained by varying the dispersion energy with respect to component 1 between $\varepsilon_{2} / \varepsilon_{1}=0.5$ and 0.95 with a decrement of 0.05 . The critical points of the highboiling component 1 and the ten different low-boiling components 2 are shown in Fig. 1 together with the vapor pressure curves of component 1 and two of the pure components 2 with the highest and lowest dispersion energy ratio $\varepsilon_{2} / \varepsilon_{1}$. The results shown in Fig. 1 were calculated with the PeTS EOS, which is known to reproduce the LJTS vapor-liquid

equilibrium very well ${ }^{5,48}$. Throughout the present study, the temperature is $T=0.77 \varepsilon k_{\mathrm{B}}^{-1}$, which corresponds to approximately 0.7 of the critical temperature of the pure component 
1 (cf. dashed line in Fig. 1).

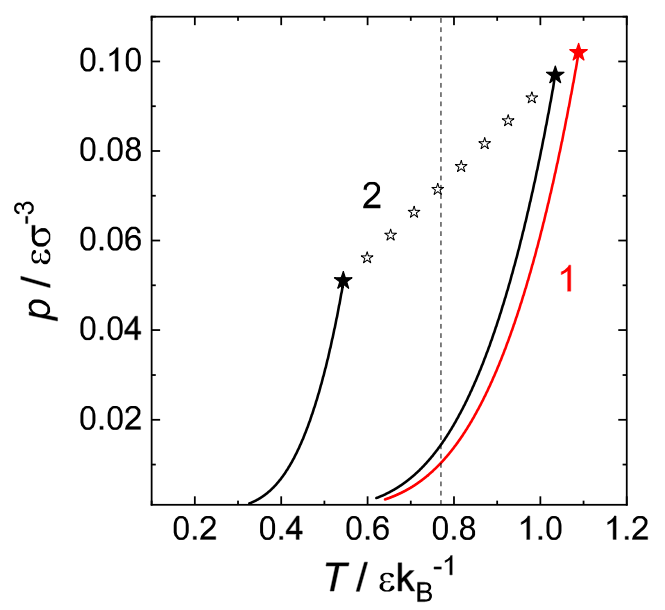

FIG. 1. Vapor pressure curves for the pure LJTS fluids that were considered in the present work. The temperature for which all simulations of the present work were carried out is $T=0.77 \varepsilon k_{\mathrm{B}}^{-1}$ and is indicated as vertical dotted line. The high-boiling component 1 is indicated in red; the different low-boiling components 2 in black. The low-boiling components 2 with lowest $\left(\varepsilon_{2} / \varepsilon_{1}=0.5\right)$ and highest $\left(\varepsilon_{2} / \varepsilon_{1}=0.95\right)$ dispersion energy used in this study are indicated as black lines. For clarity only two out of the 10 low-boiling components vapor pressure curves are shown. Stars indicate the critical points of all 11 investigated pure substances. The empty stars indicate the critical points of the low-boiling fluids with $\varepsilon_{2} / \varepsilon_{1}=0.55,0.6, . .0 .85,0.9$.

The modified Lorentz-Berthelot combination rules were employed ${ }^{49,76}$ for describing the interactions between unlike LJTS particles:

$$
\begin{aligned}
& \sigma_{12}=\frac{\sigma_{1}+\sigma_{2}}{2}, \\
& \varepsilon_{12}=\xi \sqrt{\varepsilon_{1} \varepsilon_{2}},
\end{aligned}
$$

where the index 1 and 2 indicate the interaction of two particles of the same component and the double index 12 the cross interaction between different components. The value of $\varepsilon_{12}$ describes the cross affinity between the two components which is systematically varied by varying $\xi$. The binary interaction parameter $\xi$ is used as adjustable parameter, which is considered to be state-independent. For the modeling of real fluid mixtures, its number is often obtained from a fit to experimental data ${ }^{77}$. The present work does not aim at reproducing the phase behavior of selected real mixtures. We are interested in studying 
the influence of the molecular interactions on the interfacial behavior of LJTS mixtures. Therefore, the binary interaction parameter is varied in the range $\xi=0.85$ and 1.25 with a decrement of 0.05. This results in 90 binary LJTS mixtures as shown in Fig. 2.

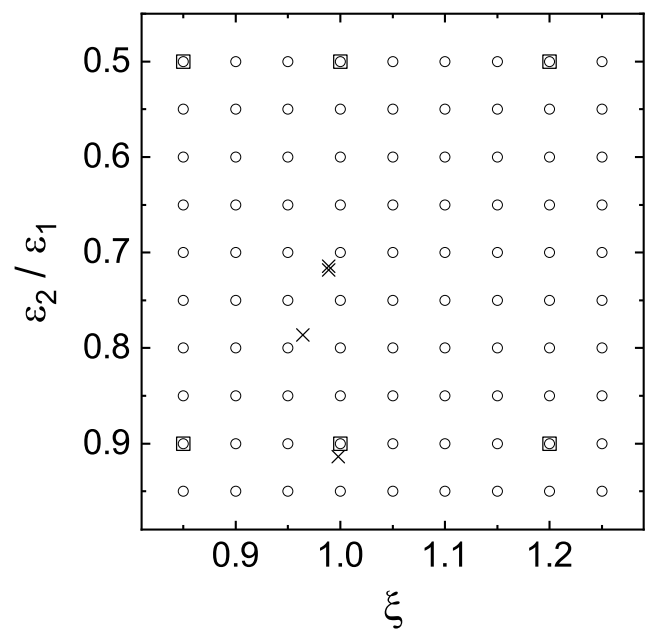

FIG. 2. Overview of the investigated binary LJTS mixtures in the plane of the binary interaction parameter $\xi$ and the ratio of the dispersion energies of the low and high-boiling component $\varepsilon_{2} / \varepsilon_{1}$. Circles indicate the 90 mixtures that were investigated in the present work. Squares mark the mixtures investigated by Stephan et al. ${ }^{5}$. The stars indicate Lennard-Jones parameters of models of real binary mixtures proposed by Vrabec et al. ${ }^{78}$ for argon-krypton, argon-methane, kryptonxenon, and methane-krypton.

The liquid phase composition is prescribed by setting $x_{2}^{\prime}=0.05 \mathrm{~mol} \mathrm{~mol}^{-1}$ throughout this study. This composition was chosen based on the experience from our previous studies, which showed that the enrichment is strongest at low concentrations of the low-boiling component in the saturated liquid phase ${ }^{1,5,8,30}$.

The binary interaction parameter $\xi$ and the ratio of the dispersion energies $\varepsilon_{2} / \varepsilon_{1}$ is not only used in the MD simulations, but the same number is also used in the PeTS EOS, and therefore also in the DGT calculations. It has been shown before that no adjustments are necessary: the phase behavior and interfacial properties that are obtained from both methods match well, when the same numbers for $\xi$ and $\varepsilon_{2} / \varepsilon_{1}$ are used in MD and DGT ${ }^{5,30}$.

Throughout the present work, all physical properties are reduced using the Lennard-Jones parameters of the high-boiling component $\varepsilon_{1}$ and $\sigma_{1}$, and the molecular mass $M$, as well as the Boltzmann constant $k_{\mathrm{B}}{ }^{64}$. 


\section{B. Molecular Simulations}

\section{Direct Vapor-Liquid Equilibrium Simulations}

In the MD simulations of vapor-liquid interfaces, systems were studied in which a liquid phase that is arranged as a slab in the middle of the simulation volume is equilibrated with two vapor phases on each side. Periodic boundary conditions were applied in all directions. The coordinate $z$ is perpendicular to the interfaces. The elongation of the simulation box normal to the interface was $80 \sigma$ and the thickness of the liquid slab in the middle of the simulation box was $40 \sigma$ to avoid finite size effects ${ }^{5,70}$. The elongation in the directions parallel to the interfaces was at least $20 \sigma$. The simulations were performed with the MD code $l s 1 \operatorname{mardyn}^{79}$ in the $N V T$ ensemble with $N=16,000$ particles. The equation of motion was solved by a leapfrog integrator ${ }^{80}$ with a time step of $\Delta t=0.001 \sigma \sqrt{M / \varepsilon}$. The equilibration was executed for 2,500,000 time steps. The production was conducted for $7,500,000$ time steps. The density and pressure profiles were computed in block averages of 500,000 time steps during the production phase. The statistical error was estimated to be three times the standard deviation of all block averages.

The vapor pressure $p^{s}$, the saturated densities $\rho^{\prime}$ and $\rho^{\prime \prime}$, and the saturated vapor and liquid phase mole fractions $x_{i}^{\prime}$ and $x_{i}^{\prime \prime}$ were computed as an average over the respective phases excluding the area close to the interface, where the first derivative of the density with respect to the $z$-coordinate significantly deviates from zero.

Also the density profiles were obtained as averages over all sampling points (blocks) in the production phase. To avoid smearing of the averaged density profiles from fluctuations of the liquid slab position in the box, the individual density profiles from one block average were shifted by setting the $z$-axis origin at $\rho_{\text {tot }}=\rho_{\text {tot }}^{\prime \prime}+0.5\left(\rho_{\text {tot }}^{\prime}-\rho_{\text {tot }}^{\prime \prime}\right)$, where $\rho_{\text {tot }}$ is the total local density $\rho_{\text {tot }}=\rho_{1}+\rho_{2}$ before averaging, as it was done in our previous works ${ }^{5,8,30}$.

The initial bulk phase compositions and number densities were estimated with the PeTS $\operatorname{EOS}^{5,48}$ to ensure that the desired value of $x_{2}^{\prime}=0.05 \mathrm{~mol} \mathrm{~mol}^{-1}$ was found in the simulations after equilibration. Further details on this procedure are given in the supplementary information.

The interfacial tension was obtained from the deviation between the normal and the 
tangential diagonal components of the overall pressure tensor ${ }^{81,82}$, i.e. the mechanical route

$$
\gamma=\frac{1}{2} \int_{-\infty}^{\infty}\left(p_{\mathrm{N}}-p_{\mathrm{T}}\right) \mathrm{d} z
$$

The normal pressure $p_{\mathrm{N}}$ is given by the $z$-component of the diagonal of the pressure tensor, and the tangential pressure $p_{\mathrm{T}}$ is determined by averaging over $x$ - and $y$-components of the diagonal of the pressure tensor. The interfacial area $S$ of each vapor-liquid interface is given by the cross section of the simulation volume normal to the $z$-axis.

\section{Henry's Law Constant Simulations}

Henry's law constants $H_{2,1}$ of the low-boiling component 2 in the solvent 1 were determined in the $N p T$ ensemble with 1372 solvent particles using Widom's test particle $\operatorname{method}^{83}$. The simulations were performed with the program $m s 2^{84}$. In the simulations, the residual chemical potential $\mu_{2}^{\infty}$ of the solute is sampled, which is directly related to the Henry's law constant by ${ }^{85}$

$$
H_{2,1}=\rho^{\prime} T \exp \left(\mu_{2}^{\infty} / T\right)
$$

where $\rho^{\prime}$ is the saturated liquid density of the solvent and $T$ is the temperature. Each simulation run consisted of 300,000 time steps equilibration and 1,000,000 time steps production.

The time step was set to $\Delta t=0.001 \sigma \sqrt{M / \varepsilon} .5200$ trial insertions were carried out per time step. The statistical uncertainty of the Henry's law constant is estimated to be three times the standard deviation of the ten block averages (the block size was 100,000 time steps).

\section{PeTS EOS and Density Gradient Theory}

\section{PeTS EOS}

The perturbed truncated and shifted (PeTS) EOS ${ }^{5,48}$ was developed to model the thermodynamic properties of the LJTS fluid. It is based on the perturbation theory of Barker and Henderson ${ }^{86}$, which splits the free energy per particle $a$ into the ideal gas contribution $a_{\text {id }}$, the free energy of a hard sphere potential $a_{\text {hs }}$ and the perturbation contribution $a_{\text {pert }}$ due to dispersion:

$$
a=a_{\mathrm{id}}+a_{\mathrm{hs}}+a_{\mathrm{pert}} .
$$

The perturbation contribution is modeled as a sum of first- and second-order contributions, i.e. $a_{\text {pert }}=a_{1}+a_{2}$, which are developed in Taylor series as functions of the packing fraction $\eta$. 
The PeTS EOS was extended to binary mixtures ${ }^{5}$ using van der Waals one-fluid theory ${ }^{53}$, which is known to perform well as long as the size parameters $\sigma_{1}$ and $\sigma_{2}$ of both components $\operatorname{are~similar~}^{87-89}$. The modified combination rules of Lorentz and Berthelot are used for the computation of the cross interaction parameters $\sigma_{12}$ and $\varepsilon_{12}$, cf. Eq. (3) and (4). The full equations of the PeTS EOS are given in Refs. ${ }^{5,48}$.

The PeTS EOS was fitted to vapor-liquid equilibrium simulation data of the LJTS fluid ${ }^{48}$. It was shown in previous work that the PeTS EOS accurately describes thermodynamic properties of the LJTS fluid both for stable and metastable states ${ }^{48}$ as well as interfacial properties $^{63}$. The PeTS EOS describes not only the pure LJTS fluid well but also LJTS mixtures $^{5}$. No adjustment of parameters is required for the accurate description of both bulk and interfacial properties of LJTS mixtures.

\section{Density Gradient Theory}

The free energy of the heterogeneous system is expanded in DGT in a Taylor series in the derivatives of the density with respect to the spatial coordinate normal to the interface $\nabla^{m} \underline{\rho}, m=1,2, .$. and truncated after the square gradient term. To model fluid interfaces, DGT only requires a model of the free energy of the bulk fluid and the so-called influence parameter $\kappa_{i j}$. The influence parameter describes the influence of the density gradients on the free energy. For a comprehensive introduction we refer to $\mathrm{s}^{3,90-92}$. For a planar interface of a binary mixture with the components $i$ and $j$, the free energy can be written as:

$$
a(\underline{\rho}, \nabla \underline{\rho})=a_{0}(\underline{\rho})+\sum_{i=1}^{2} \sum_{j=1}^{2} 1 / 2 \kappa_{i j} \nabla \rho_{i} \nabla \rho_{j},
$$

where $a_{0}(\underline{\rho})$ is the free energy of the homogeneous system at the local composition and $\rho$ indicates the vector of the number densities $\rho_{i}$ and $\nabla \rho$ the vector of the corresponding gradients. $\kappa_{i j}$ are the influence parameters, for the pure substances (if $i=j$ ) and the cross interaction (if $i \neq j$ ). In the case of $i=j$, indices are labeled only with one subscript for simplicity in the following, e.g. $\kappa_{11}=\kappa_{1}$. The PeTS EOS is employed for calculating the free energy in Eq. (8).

The cross-interaction influence parameter was assumed here to be the geometric mean of the pure substance influence parameters ${ }^{93,94}$

$$
\kappa_{i j}=\sqrt{\kappa_{i} \kappa_{j}}
$$

In a previous work of our group it was shown that this assumption leads to almost perfect 
agreement between results from DGT and computer experiment ${ }^{5}$ for LJTS mixtures in the entire composition range. The influence parameter of the high-boiling component was adopted from Ref. ${ }^{48}$, i.e. $\kappa_{1}=2.7334 \sigma^{5} \varepsilon$. The influence parameters of the low-boiling

component 2 were calculated by $\kappa_{2}=\kappa_{1} \frac{\varepsilon_{2}}{\varepsilon_{1}}$. The results for the interfacial properties of mixtures are thereby predictions.

To obtain the equilibrium density profiles $\rho_{i}(z)$ of both components connecting the bulk phases, Eq. (8) has to be minimized. For solving the DGT equation for both components in the interfacial domain, we used the stabilized DGT algorithm proposed by Mu et al. ${ }^{95}$, which is numerically robust and avoids choosing a reference component, which has numerical pitfalls $^{96}$. The domain length is set to $20 \sigma$ with a spatial discretization of $0.02 \sigma$.

The surface tension $\gamma$ was calculated by

$$
\gamma=\int \sum_{i=1}^{2} \sum_{j=1}^{2} \kappa_{i j} \nabla \rho_{i} \nabla \rho_{j} \mathrm{~d} z .
$$

\section{Interfacial Properties}

The MD simulations as well as the DGT yield the component density profiles $\rho_{i}(z)$ of both components $i=1,2$ in the interfacial region. On the basis of the density profiles $\rho_{1}(z)$ and $\rho_{2}(z)$, the relative adsorption of the low-boiling component as defined by Gibbs ${ }^{97}$ can be computed by the symmetric interface segregation according to Telo da Gama and Evans ${ }^{98,99}$

$$
\Gamma_{2}^{(1)}=-\left(\rho_{2}^{\prime}-\rho_{2}^{\prime \prime}\right) \int_{-\infty}^{\infty}\left[\frac{\rho_{1}(z)-\rho_{1}^{\prime}}{\rho_{1}^{\prime}-\rho_{1}^{\prime \prime}}-\frac{\rho_{2}(z)-\rho_{2}^{\prime}}{\rho_{2}^{\prime}-\rho_{2}^{\prime \prime}}\right] \mathrm{d} z
$$

where $\rho_{1}^{\prime}, \rho_{1}^{\prime \prime}$ and $\rho_{2}^{\prime}, \rho_{2}^{\prime \prime}$ are the component densities at saturation in the bulk liquid and bulk vapor phase, respectively. $\Gamma_{2}^{(1)}$ is the relative surface adsorption of component 2 at component 1.

The interfacial excess can also be characterized by the so-called enrichment $E_{i}{ }^{1}$ which is defined as the ratio of the maximum local density of component $i$ in the interfacial region and the larger of the corresponding densities in the two bulk phases. Hence, the enrichment of the low-boiling component 2 is

$$
E_{2}=\frac{\max \left(\rho_{2}(z)\right)}{\max \left(\rho_{2}^{\prime}, \rho_{2}^{\prime \prime}\right)} .
$$

The relative adsorption and the enrichment are linked, but do not contain the same information $^{1,5}$. Adsorption may occur although no enrichment $\left(E_{2}=1\right)$ is present, for 
example if the two component density profiles are shifted relative to each other ${ }^{5}$. But an enrichment will generally result in an adsorption.

To describe the thickness of the planar interface, we use the 90-10\% definition for the effective interfactial thickness $L_{10}^{90}$ according to Lekner and Henderson ${ }^{100}$, which is the distance between the points where the total local density $\rho_{\text {tot }}(z)=\rho_{1}(z)+\rho_{2}(z)$ reaches $10 \%$ and $90 \%$ of the total bulk number densities, respectively:

$$
\begin{aligned}
& L_{10}^{90}=z\left(\rho_{90}^{\mathrm{tot}}\right)-z\left(\rho_{10}^{\mathrm{tot}}\right), \\
& \rho_{10}^{\mathrm{tot}}=\rho_{\mathrm{tot}}^{\prime \prime}+0.1\left(\rho_{\mathrm{tot}}^{\prime}-\rho_{\mathrm{tot}}^{\prime \prime}\right), \\
& \rho_{90}^{\mathrm{tot}}=\rho_{\mathrm{tot}}^{\prime \prime}+0.9\left(\rho_{\mathrm{tot}}^{\prime}-\rho_{\mathrm{tot}}^{\prime \prime}\right) .
\end{aligned}
$$

The origin on the $z$-axis of interfacial profiles from both MD and DGT was chosen such that $\rho_{\text {tot }}(z)=0$ at $\rho_{\text {tot }}=\rho_{\text {tot }}^{\prime \prime}+0.5\left(\rho_{\text {tot }}^{\prime}-\rho_{\text {tot }}^{\prime \prime}\right)$.

\section{RESULTS AND DISCUSSION}

\section{A. Vapor-Liquid Equilibria}

The vapor-liquid equilibrium of LJTS mixtures (cf. Fig. 2) was investigated at $T=$ $0.77 \varepsilon k_{\mathrm{B}}^{-1}$ with both MD simulations and the PeTS EOS. The MD simulations were carried out for a liquid phase mole fraction of the low-boiling component 2 of $x_{2}^{\prime}=0.05 \mathrm{~mol} \mathrm{~mol}^{-1}$. The numerical values of the VLE data at $x_{2}^{\prime}=0.05 \mathrm{~mol} \mathrm{~mol}^{-1}$ and $T=0.77 \varepsilon k_{\mathrm{B}}^{-1}$ obtained from MD and the PeTS EOS are reported for all 90 mixtures in the supplementary material (vapor pressure, gas phase composition, and saturated densities). The PeTS EOS was used to calculate the isothermal phase diagrams of all 90 mixtures. Representative results for 30 systems are shown in Fig. 3. For all mixtures the high-boiling component 1 is the same. Its vapor pressure is $p^{s}=0.0104 \varepsilon \sigma^{-3}$ at $T=0.77 \varepsilon k_{\mathrm{B}}^{-1}$. The low-boiling component 2 in

the mixtures varies. Results for different values of $\varepsilon_{2} / \varepsilon_{1}$ are shown in the different rows of the matrix depicted in Fig. 3. For high values of $\varepsilon_{2} / \varepsilon_{1}$, the component 2 is subcritical, for small values of $\varepsilon_{2} / \varepsilon_{1}$, it is supercritical. The critical temperature of the pure component 2 is $T_{\mathrm{c}}=0.77 \varepsilon k_{\mathrm{B}}^{-1}$ for $\varepsilon_{2} / \varepsilon_{1}=0.71$. In the columns of the matrix shown in Fig. 3, results for different choices of $\xi$ are presented. For $\xi=1$ the mixtures are almost ideal in the sense of Raoult's law. For $\xi>1$ attractive interactions prevail and the mixtures tend to 
form high-boiling azeotropes, while for $\xi<1$ repulsive interactions prevail and the mixtures tend to form low-boiling azeotropes. Decreasing $\xi$ below the lowest value that was studied in the present work $(\xi=0.85)$ leads to a liquid-liquid phase split at $T=0.77 \varepsilon k_{\mathrm{B}}^{-1}$. As studying liquid-liquid equilibria was out of the scope of the present work, only mixtures with $\xi>0.85$ were studied. The critical pressures of the mixtures with small values of $\varepsilon_{2} / \varepsilon_{1}$ show an interesting behavior: they remain almost constant upon lowering $\xi$ and increase only sharply for the lowest value of $\xi$ shown in Fig. 3.

Qualitatively, the bubble lines of the mixtures in the $\xi, \varepsilon_{2} / \varepsilon_{1}$-plane differ more than the dew lines. This can be interpreted as a consequence of the fact that the interactions in the liquid phase are dominating the VLE behavior compared to a minor influence of the vapor phase. This is evident, since the self interactions and cross interactions have a stronger impact in the dense liquid phase.

The results for the Henry's law constants of all 90 investigated mixtures at $T=0.77 \varepsilon k_{\mathrm{B}}^{-1}$ that were obtained form the MD simulations as well as from the PeTS EOS are shown in Fig. 4 as a function of the binary interaction parameter $\xi$ and the ratio of the dispersion energies $\varepsilon_{2} / \varepsilon_{1}$. The numeric values are reported in the supplementary material. The results from the computer experiment and the theory are found to be in good agreement for all mixtures. As expected, the Henry's law constant $H_{2,1}$ increases steadily with decreasing ratio of dispersion energies $\varepsilon_{2} / \varepsilon_{1}$ as well as with decreasing values of $\xi$.

The plot of $H_{2,1}$ over $\varepsilon_{2} / \varepsilon_{1}$ and $\xi$ shown in Fig. 4 has some interesting features. First, moving along the diagonal connecting the corner points $\left(\varepsilon_{2} / \varepsilon_{1}=0.5, \xi=0.85\right.$, labeled here as corner $A)$ and $\left(\varepsilon_{2} / \varepsilon_{1}=0.95, \xi=1.25\right.$, labeled here as corner $\left.B\right)$, the Henry's law constant first increases only weakly but then steeply. This is due to the fact that towards corner $B$ the mean dispersive interactions in the liquid phase become less attractive, which decreases the solubility of the low-boiling component 2 in the high-boiling component 1.

Furthermore, the surface $H_{2,1}=H_{2,1}\left(\varepsilon_{2} / \varepsilon_{1}, \xi\right)$ has a remarkably simple shape. The traces of the level curves in the $\varepsilon_{2} / \varepsilon_{1}, \xi$-plane can be approximated by segments of root functions (cf. Fig. 4), which results in an approximately radial symmetry of the function $H_{2,1}=H_{2,1}\left(\varepsilon_{2} / \varepsilon_{1}, \xi\right)$ in the investigated range of $\varepsilon_{2} / \varepsilon_{1}$ and $\xi$. As only a segment of the function is plotted in Fig. 4, the curvature can only be seen by inspecting the traces of the isolines. Going from corner $A$ to corner $B$ in a straight line follows the gradient of that function. 


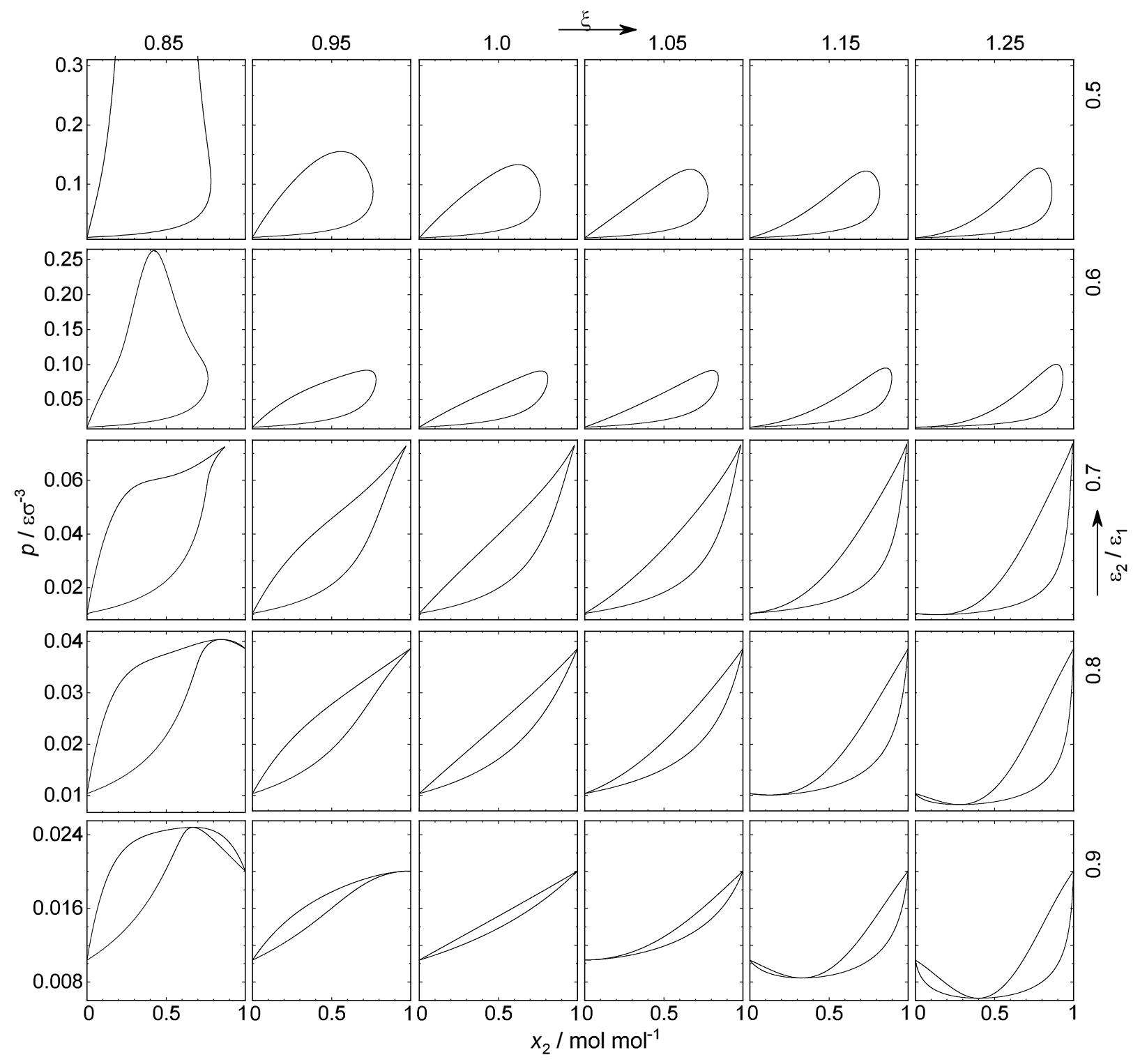

FIG. 3. Selection of isothermal $p-x$ phase diagrams of binary LJTS mixtures at $T=0.77 \varepsilon k_{\mathrm{B}}^{-1}$ for different binary interaction parameter $\xi$ and ratio of the dispersion energies of both components $\varepsilon_{2} / \varepsilon_{1}$. The phase diagrams were calculated by the PeTS EOS ${ }^{5,48}$. Columns have constant binary interaction parameter as indicated at the top; rows have constant ratio of the dispersion energies as indicated on the right. Corner $A$ is top left, corner $B$ bottom right, see text.

Fig. 5 shows the difference of the number density of the low-boiling component between the bulk vapor and bulk liquid phase $\Delta \rho_{2}=\rho_{2}^{\prime}-\rho_{2}^{\prime \prime}$ at $x_{2}^{\prime}=0.05 \mathrm{~mol} \mathrm{~mol}^{-1}$ and $T=$ $0.77 \varepsilon k_{\mathrm{B}}^{-1}$ as a function of the binary interaction parameter $\xi$ and the ratio of the dispersion energies $\varepsilon_{2} / \varepsilon_{1}$. The difference of the number density of the low-boiling component $\Delta \rho_{2}$ was 


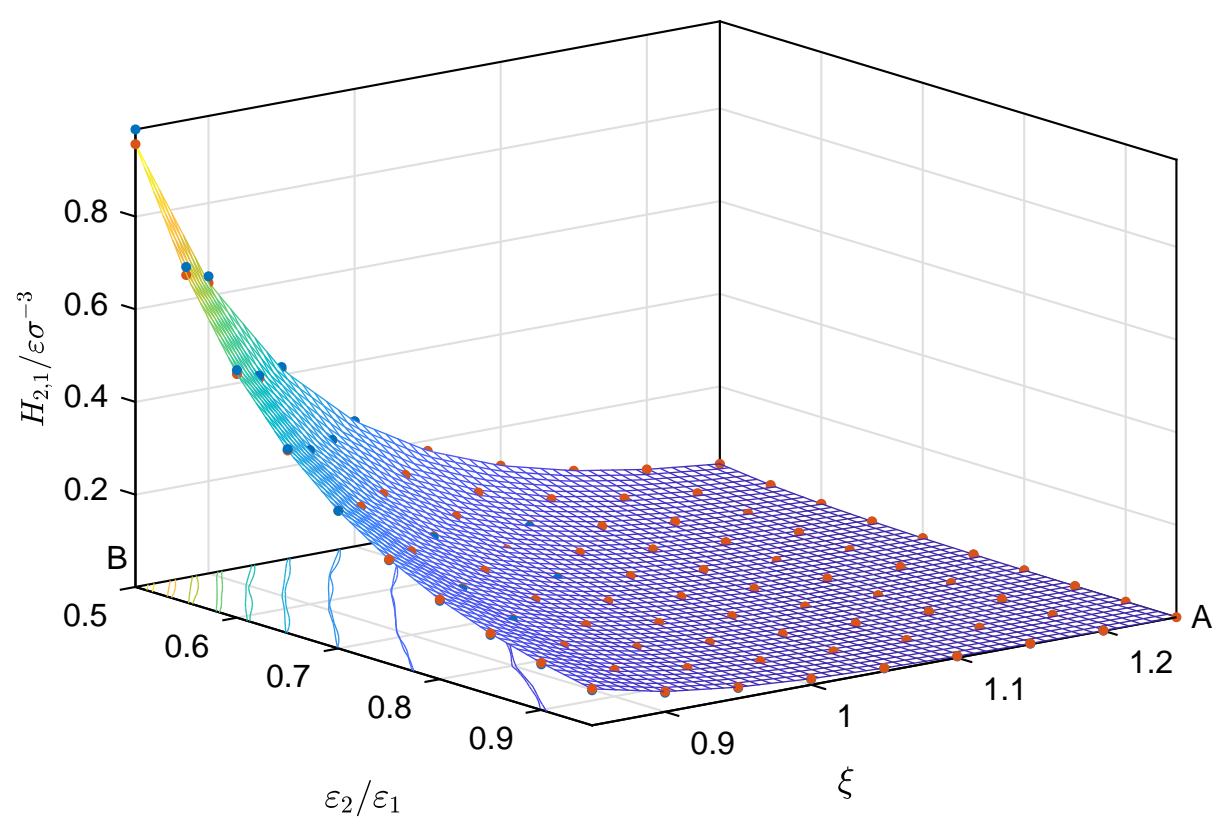

FIG. 4. Henry's law constant $H_{2,1}$ of binary LJTS mixtures $1+2$ at $T=0.77 \varepsilon k_{\mathrm{B}}^{-1}$ as a function of the binary interaction parameter $\xi$ and the ratio of the dispersion energies $\varepsilon_{2} / \varepsilon_{1}$. Results from MD: blue points; results from PeTS EOS ${ }^{5,48}$ : orange points. The surface is a linear interpolation of the EOS results. The color of the surface is coded by its height.

calculated for all 90 LJTS mixtures using both MD simulation and the PeTS EOS. The computer experiments and the EOS have a distinct offset which vanishes in corner B, but the qualitative behavior of the results from both methods agrees well.

The deviations show that the VLE behavior of LJTS mixtures is not described perfectly by the PeTS EOS, but in assessing the deviations it has to be considered that $\Delta \rho_{2}$ is a very sensitive property. The same symmetry as for the Henry's law constant (cf. Fig. 4 ) is also found for $\Delta \rho_{2}\left(\varepsilon_{2} / \varepsilon_{1}, \xi\right)$ (Fig. 5) but the trends are inverse: $\Delta \rho_{2}$ is low where $H_{2,1}$ is high. This is not unexpected since both properties express the tendency of the low-boiling component to portion on both phases.

An interesting feature of $\Delta \rho_{2}\left(\varepsilon_{2} / \varepsilon_{1}, \xi\right)$ is the zero crossing line of $\Delta \rho_{2}$ close to corner $B$. In a wide range of investigated mixtures $\Delta \rho_{2}>0$, i.e. the the low-boiling component accumulates in the liquid phase. Vice versa, for $\Delta \rho_{2}<0$ the low-boiling component has a preferential residency in the vapor phase. 


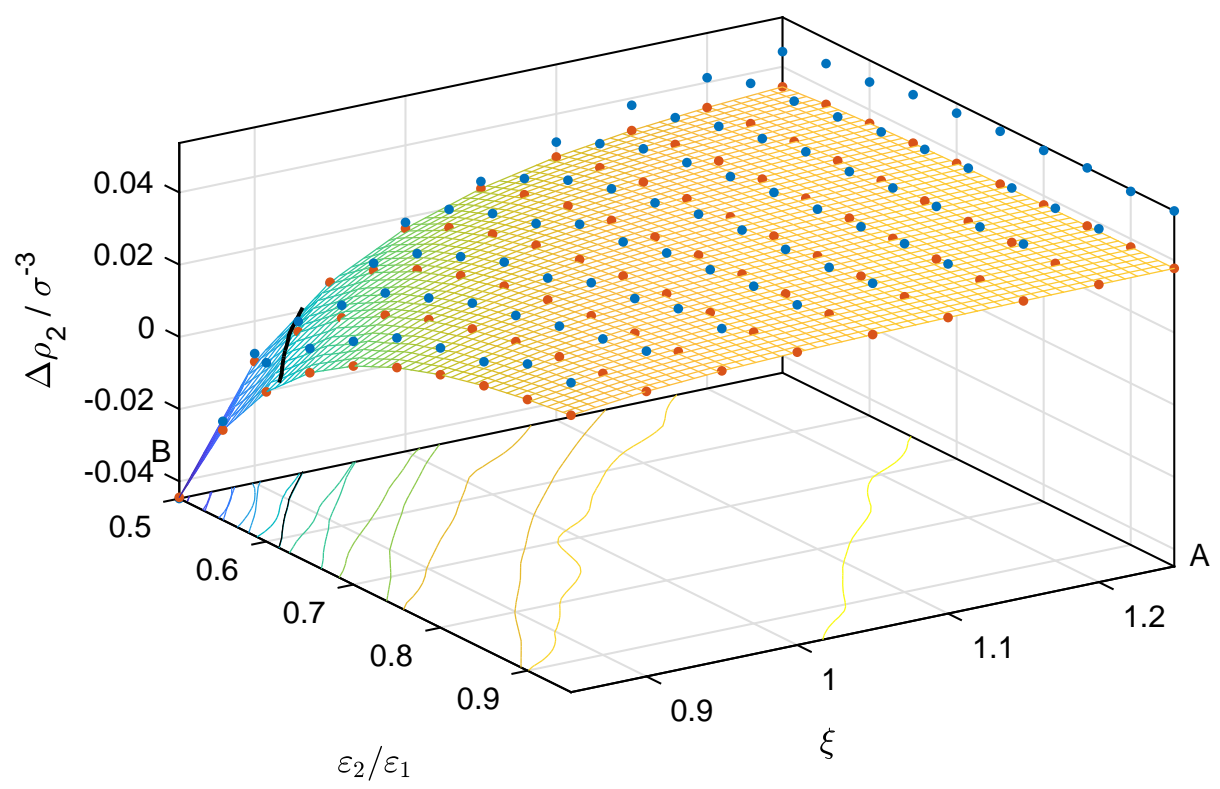

FIG. 5. Difference in the bulk number density of the low-boiling component $\Delta \rho_{2}=\rho_{2}^{\prime}-\rho_{2}^{\prime \prime}$ at $T=0.77 \varepsilon k_{\mathrm{B}}^{-1}$ and $x_{2}^{\prime}=0.05 \mathrm{~mol} \mathrm{~mol}^{-1}$ as a function of the binary interaction parameter $\xi$ and the ratio of the dispersion energies $\varepsilon_{2} / \varepsilon_{1}$. Results from MD: blue points; results from PeTS $\operatorname{EOS}^{5,48}$ : orange points. The surface is a linear interpolation of the EOS results. The color of the surface is coded by its height position. The black line indicates the trace of the zero crossing line $\Delta \rho_{2}=0$ from the EOS results.

\section{B. Vapor-Liquid Interfaces}

Planar vapor-liquid interfaces were investigated by both MD and DGT at $T=0.77 \varepsilon k_{\mathrm{B}}^{-1}$ and $x_{2}^{\prime}=0.05 \mathrm{~mol} \mathrm{~mol}^{-1}$. Besides the density profiles, the interfacial enrichment (cf. Eq. (12)), relative adsorption (cf. Eq. (11)), interfacial tension (cf. Eq. (10) and (5)), and interfacial thickness (cf. Eq. (13)) were investigated for all 90 binary LJTS mixtures (cf. Fig. 2). Density profiles are shown in Fig. 6, the enrichment in Fig. 7, the relative adsorption in Fig. 8, the surface tension in Fig. 9, and the interfacial thickness in Fig. 10.

The prescribed liquid phase composition is exactly matched in DGT, but not by MD due to fluctuations. The mole fraction of the low-boiling component in the liquid phase obtained from the MD simulations are in the range $x_{2}^{\prime}=0.05 . .0 .07 \mathrm{~mol} \mathrm{~mol}^{-1}$. The numeric values of the interfacial properties from both MD and DGT as well as the actual liquid phase composition in MD are reported in Table I. The error bars of the MD simulation results are not shown in the plots in Figs. 7 - 10 for clarity, but the statistical uncertainties are 
reported in Table $\mathrm{I}$.

\section{Density Profiles and Enrichment}

Selected results for the density profiles are shown in Fig. 6. The plot is organized in the same way as Fig. 3, i.e. the selected systems are the same. The density profiles are plotted as a function of the spatial coordinate $z$ normal to the planar interface. For clarity, only the DGT density profiles are shown here. It was already shown by Stephan et al. ${ }^{5}$ that the density profiles of LJTS mixtures predicted by MD and DGT+PeTS agree very well. This is confirmed in the present work. It can be seen from Fig. 6 that the enrichment of the low-boiling component $E_{2}$, i.e. the relative hight of the peak in $\rho_{2}(z)$, generally increases with decreasing $\varepsilon_{2} / \varepsilon_{1}$ and decreasing $\xi$. Details become clearer in Fig. 7 , where $E_{2}$ is plotted as a function of $\varepsilon_{2} / \varepsilon_{1}$ and $\xi$. The layout of the plot is similar to that in Figs. 4 and 5 .

In contrast to the density profile of the low-boiling component 2, which dramatically changes in the $\xi, \varepsilon_{2} / \varepsilon_{1}$-plane, the density profile of the high-boiling component 1 remains fairly similar among the different mixtures, as already discussed in our previous work ${ }^{5}$. No enrichment of the high-boiling component 1 is observed in any mixture.

Starting in corner $A$, no enrichment of the low-boiling component 2 is found. The enrichment increases with a decreasing $\Delta \rho_{2}$ towards the corner $B$ (cf. Fig. 5). The density profiles in the region of the $\xi, \varepsilon_{2} / \varepsilon_{1}$-plane where $\Delta \rho_{2}$ is close to zero show a particularly high enrichment. A significant enrichment of the low-boiling component is found for mixtures that exhibit a low solubility of the low-boiling component in component 1, i.e. large Henry's law constants (cf. Fig. 4).

The predictions for the enrichment $E_{2}$ from the computer experiments and the theory are found to be in good qualitative agreement for all investigated mixtures. DGT overestimates the enrichment predicted by MD for mixtures close to corner B. Such an overestimation of the enrichment $E_{2}$ by DGT compared to MD results was also reported previously by Becker et al. ${ }^{1}$ and Stephan et al. ${ }^{5}$ for mixtures where the low-boiling component is supercritical. The enrichment predicted by MD in corner $A$ is slightly above unity, while DGT predicts no enrichment, i.e. $E_{2}=1$, in that region. As already discussed in Ref. ${ }^{5}$, the deviation of the MD results from $E_{2}=1$ is attributed to the fluctuations in connection with the definition of the enrichment (cf. Eq. (12)). 


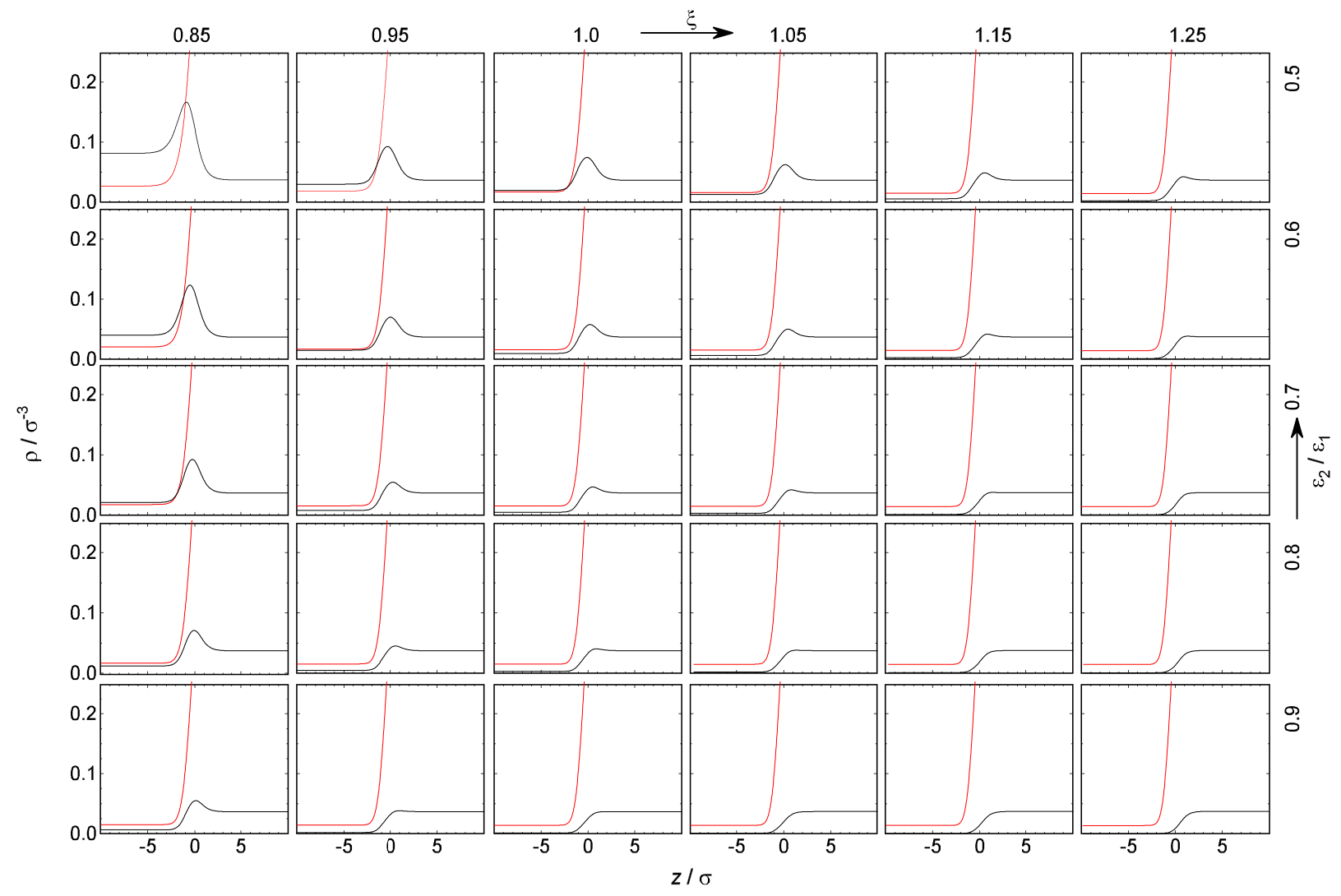

FIG. 6. Selection of density profiles at planar vapor-liquid interfaces calculated by DGT: local density of the low-boiling component 2 (black line) and high-boiling component 1 (red line) as a function of the spatial coordinate $z$ normal to the interface in the plane of the binary interaction parameter $\xi$ and the ratio of the dispersion energies $\varepsilon_{2} / \varepsilon_{1}$. Columns have constant binary interaction parameter as indicated on the top; rows have constant ratio of the dispersion energies as indicated on the right. The temperature is always $T=0.77 \varepsilon k_{\mathrm{B}}^{-1}$ and the liquid phase concentration is $x_{2}^{\prime}=0.05 \mathrm{~mol} \mathrm{~mol}^{-1}$. Corner $A$ is top left, corner $B$ bottom right, see text.

Again, the same symmetry as in the plots shown in Figs. 4 and 5 (Henry's law constant $H_{2,1}$ and density difference $\left.\Delta \rho_{2}\right)$ is also found for $E_{2}\left(\varepsilon_{2} / \varepsilon_{1}, \xi\right)$.

In the corner $A$ (high mean dispersive interactions), practically no enrichment $E_{2}$ is found, while strong enrichment is found in corner B. Starting in corner $A$ and going to corner $B$ on a straight line, the enrichment $E_{2}$ remains around unity for a while, and then increases sharply. Interestingly, there is a drop in $E_{2}$, before corner $B$ is reached.

The position of the line of the maximal enrichment $E_{2}$ in the $\xi, \varepsilon_{2} / \varepsilon_{1}$-plane, as indicated in Fig. 7, is in very good agreement with the zero crossing line of the difference of the 
number density of component $2 \Delta \rho_{2}=0$, cf. Fig. 5. I.e. it corresponds to mixtures for which the density of the component 2 is equal in both phases. For mixtures on that line, any adsorption of the low-boiling component at the interface must result in an enrichment for geometric reasons. On the contrary, for mixtures with $\Delta \rho_{2} \neq 0$ a relative adsorption can also arise from a relative shift of $\rho_{2}(z)$ with respect to $\rho_{1}(z)$. Even though the predicted magnitude of the enrichment by MD and DGT differs slightly, the position of the maximum line in the $\xi, \varepsilon_{2} / \varepsilon_{1}$-plane agrees perfectly.

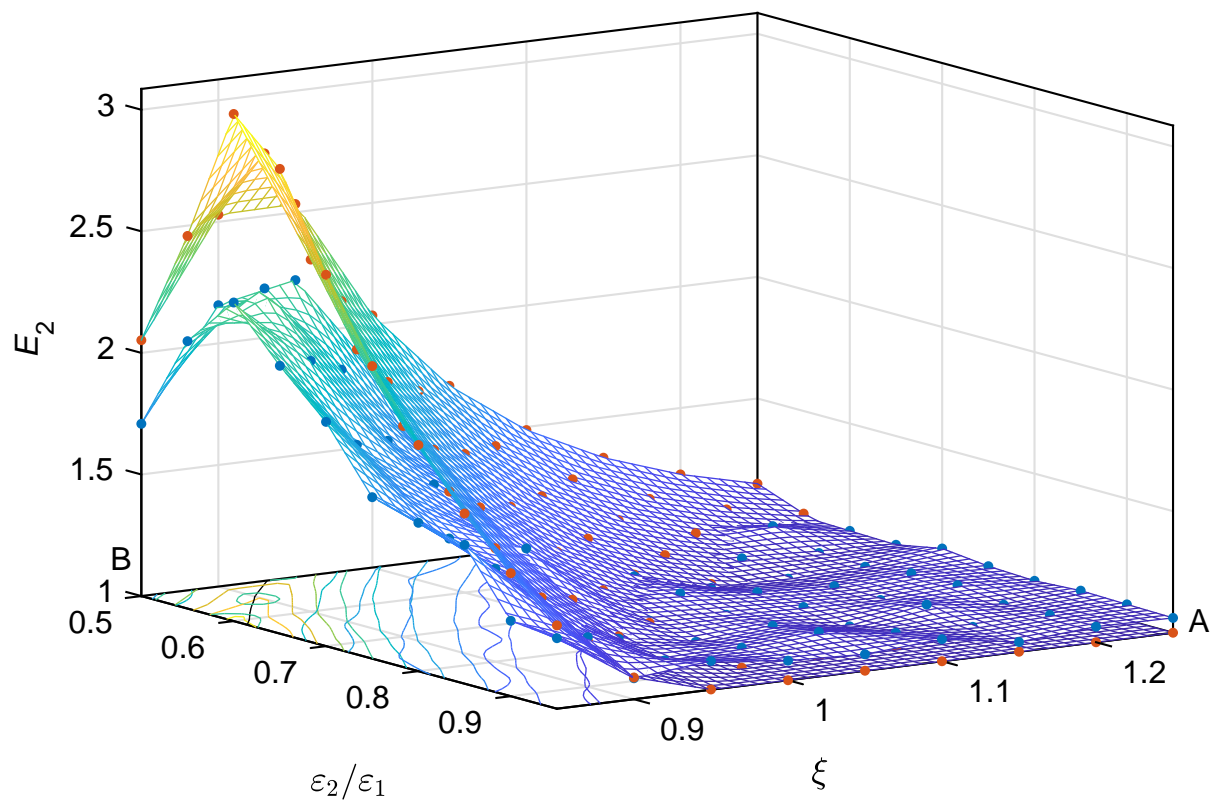

FIG. 7. Enrichment $E_{2}$ of the low-boiling component 2, as defined by Eq. (12), at the interface as a function of the binary interaction parameter $\xi$ and the ratio of the dispersion energies $\varepsilon_{2} / \varepsilon_{1}$ at $T=0.77 \varepsilon k_{\mathrm{B}}^{-1}$ and $x_{2}^{\prime}=0.05 \mathrm{~mol} \mathrm{~mol}^{-1}$. Results from MD: blue points; results from DGT: orange points. The surfaces are linear interpolations of the respective results. The color of the surfaces is coded by its height position. The black line indicates the trace of the zero crossing line $\Delta \rho_{2}=0$ from the DGT results, cf. Fig. 5. The corners $A$ and $B$ to which the text refers are indicated.

\section{Relative Adsorption}

Fig. 8 shows the relative adsorption $\Gamma_{2}^{(1)}$ of the low-boiling component at the vaporliquid interface, as defined by Eq. (11), as a function of the binary interaction parameter $\xi$ and the ratio of the dispersion energies $\varepsilon_{2} / \varepsilon_{1}$. Again results from both MD and DGT are 
shown. The predictions from the computer experiment and the theory are found to be in good agreement for all investigated mixtures. The MD results show some scattering due to fluctuations in the simulations, while results from the theory are smooth. The symmetry that was observed in the previous plots of this type is also observed here. In contrast to the enrichment $E_{2}$, the relative adsorption increases steadily when going from corner $A$ to corner $B$. The relative adsorption of the low-boiling component 2 is negative close to the corner $A$, i.e. high mean attractive interactions, and positive elsewhere. The zero crossing line of the relative adsorption (black line in Fig. 8) is roughly identical with the line that separates mixtures with high-boiling azeotropes from zeotropic mixtures, cf. Fig. 3. Hence, the relative adsorption $\Gamma_{2}^{(1)}$ is negative for mixtures with high-boiling azeotropes, while it is positive for other mixtures, which reinforces findings for the relative adsorption in Ref. ${ }^{5,39,101-103}$.

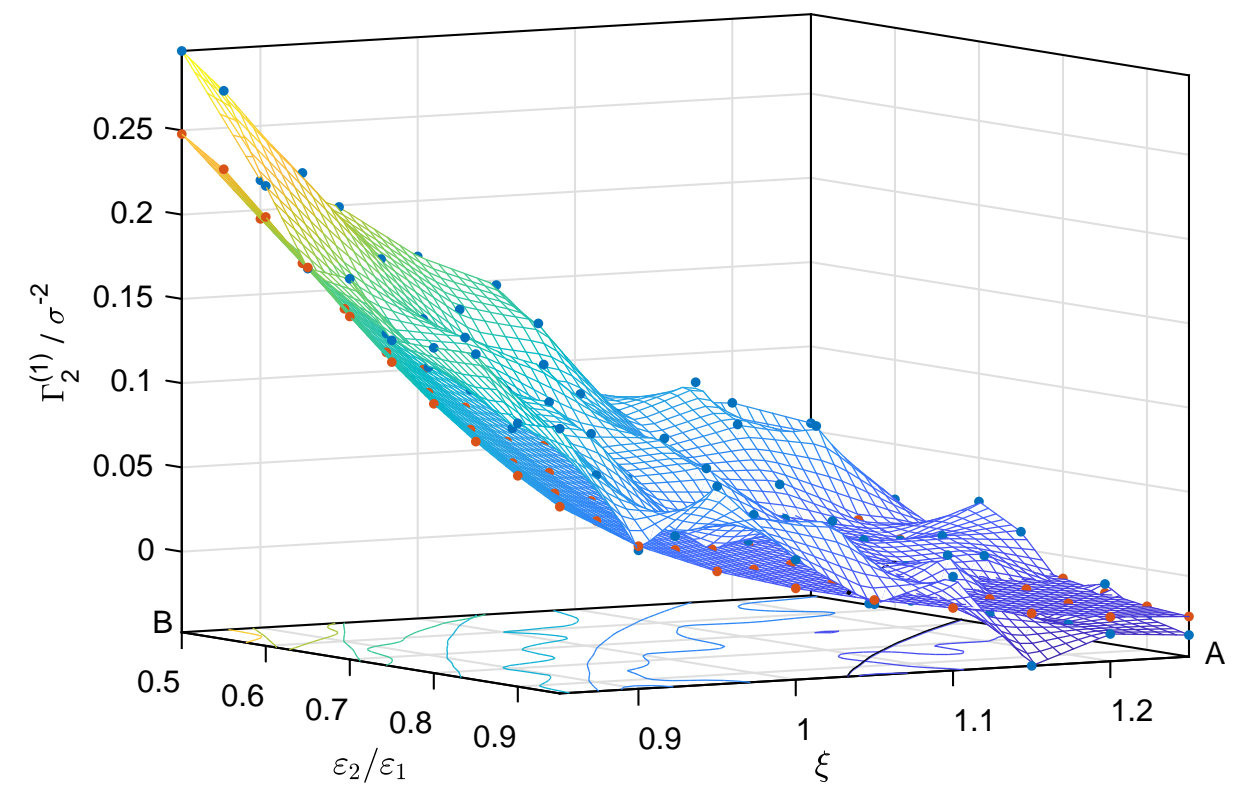

FIG. 8. Relative adsorption $\Gamma_{2}^{(1)}$ of the low-boiling component 2, as defined by Eq. (11), at the interface as a function of the binary interaction parameter $\xi$ and the ratio of the dispersion energies $\varepsilon_{2} / \varepsilon_{1}$ at $T=0.77 \varepsilon k_{\mathrm{B}}^{-1}$ and $x_{2}^{\prime}=0.05 \mathrm{~mol} \mathrm{~mol}^{-1}$. Results from MD: blue points; results from DGT: orange points. The surfaces are linear interpolations of the respective results. The color of the surfaces is coded by its height position. The black line indicates the trace of the zero crossing line $\Gamma_{2}^{(1)}=0$ from the DGT results.

Furthermore, the relative adsorption dependency on $\xi$ and $\varepsilon_{2} / \varepsilon_{1}$ can be split into two characteristic regions: one region exhibiting a non-linear regime and one region exhibiting 
a linear regime. Going from corner $A$ to corner $B$, the relative adsorption exhibits first a convex increase, whereas approaching corner $B, \Gamma_{2}^{(1)}$ increases linearly. This can only be seen in the results obtained from DGT, since the MD results are superimposed by significant scattering. The breakup between the linear and non-linear regime of $\Gamma_{2}^{(1)}$ corresponds well with the region where the enrichment of the low-boiling component is close to unity and the region of significant enrichment. This indicates again ${ }^{1,5}$, that the enrichment and the relative adsorption contain different information and it is emphasized that both should be discussed. Relative adsorption may occur even if there is no enrichment, but an enrichment will in general result in an adsorption.

\section{Surface Tension}

Fig. 9 shows the surface tension as a function of the binary interaction parameter $\xi$ and the ratio of dispersion energies $\varepsilon_{2} / \varepsilon_{1}$. The results from both MD and DGT are shown. The predictions from the computer experiments and the theory agree remarkably well for all investigated mixtures, i.e. the DGT results mostly lie within the MD error bars (cf. Table I). Only slight systematic deviations are found in corner B, where the DGT results overestimate those from computer experiments. The same type of symmetry as for the properties discussed above (cf. Figs. 4, 5, 7,8), is also observed for $\gamma\left(\varepsilon_{2} / \varepsilon_{1}, \xi\right)$.

Going from corner $A$ to corner $B$, the surface tension decreases steadily. It is found that the line, for which the surface tension of the mixture equals the surface tension of the pure component 1 (cf. Fig, 9) is in good agreement with the line for which the relative adsorption is zero (cf. Fig. 8). This is related to the Gibbs adsorption equation which relates the surface excess to the concentration dependence of the surface tension. Adding component 2 to the pure component 1 in corner $A$, i.e. the region of mixtures with the highest mean dispersive interactions, leads to an increase of the surface tension. This is a typical finding for mixtures with a high-boiling azeotrope $\mathrm{e}^{5,39,104}$. For mixtures with a low-boiling azeotrope

on the other hand, adding component 2 usually results in a decreased surface tension ${ }^{5,39,104}$, which is also found in our data. 


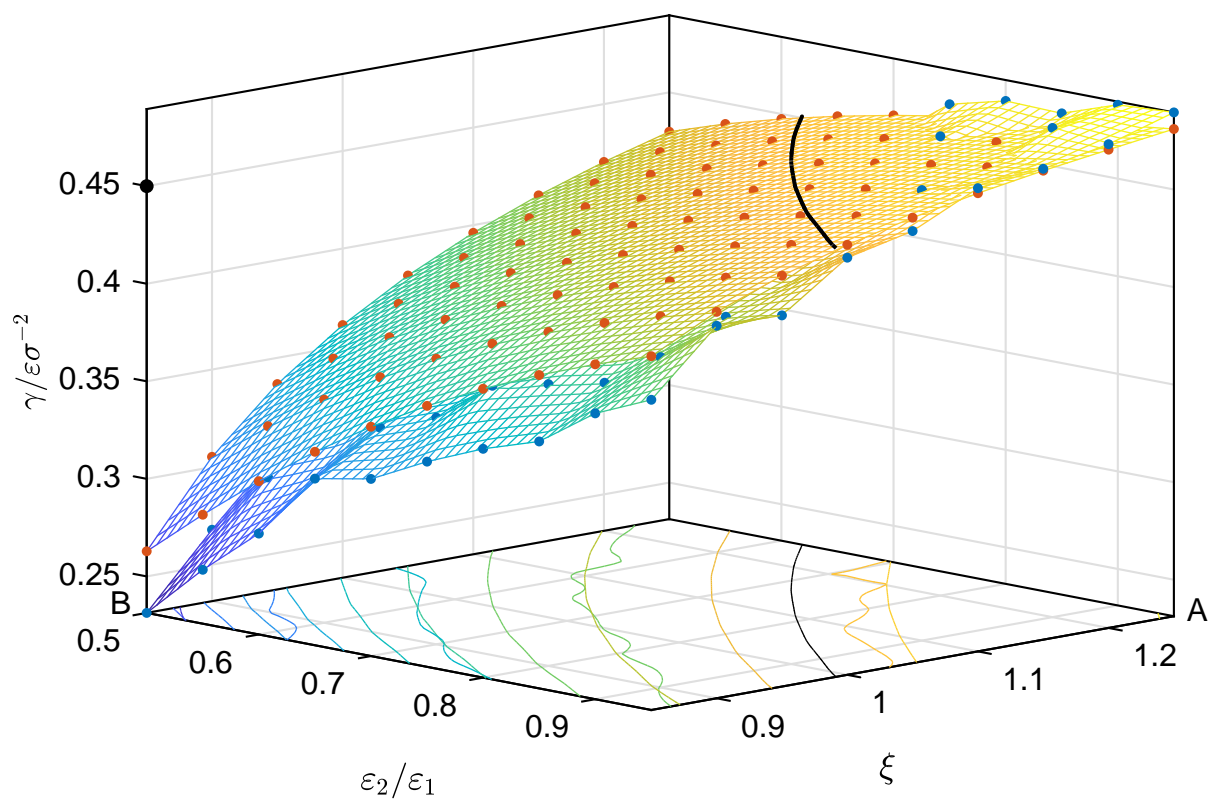

FIG. 9. Interfacial tension $\gamma$, as defined by Eq. (10) and (5), as a function of the binary interaction parameter $\xi$ and the ratio of the dispersion energies $\varepsilon_{2} / \varepsilon_{1}$ at $T=0.77 \varepsilon k_{\mathrm{B}}^{-1}$ and $x_{2}^{\prime}=0.05 \mathrm{~mol} \mathrm{~mol}^{-1}$. Results from MD: blue points; results from DGT: orange points. The surfaces are linear interpolations of the respective results. The color of the surfaces is coded by its height position. The black dot indicates the surface tension $\gamma_{1}$ of the pure component 1 at $T=0.77 \varepsilon k_{\mathrm{B}}^{-1}$ according to Stephan et al. ${ }^{63}$. The black line indicates the crossing line of $\gamma=\gamma_{1}$ from the DGT results.

\section{Interfacial Thickness}

Fig. 10 shows the interfacial thickness $L_{10}^{90}$ as defined by Eq. (13) as a function of the binary interaction parameter $\xi$ and the ratio of dispersion energies $\varepsilon_{2} / \varepsilon_{1}$ for both MD and DGT. The predictions from the computer experiments and the theory agree qualitatively very well, but are shifted significantly. The interfacial thickness from DGT is systematically lower than that from the computer experiments. This could be caused by fluctuations that are present in MD but not in $\mathrm{DGT}^{1,5,63}$, but it could also be caused by differences between the PeTS EOS and the LJTS force field. The latter hypothesis is supported by the fact that in a recent study, no such differences were observed for the LJ fluid ${ }^{30}$.

On the average, the DGT underestimates the MD interfacial thickness by approximately $-15 \%$, which is in line with results reported earlier for the corresponding pure substances ${ }^{63}$ 
as well as for selected LJTS mixtures in the entire composition range ${ }^{5}$.

Again, a symmetric behavior is found for $L_{10}^{90}\left(\varepsilon_{2} / \varepsilon_{1}, \xi\right)$. Going from corner $A$ to corner $B$, the interfacial thickness increases steadily. This is in line with the decay of the surface tension, since broader and smoother density profiles result in a lower surface tension. The interfacial thickness is almost twice as high in corner $B$ as in corner $A$.

The comparison of Figs. 4, 6 - 10 shows that all investigated bulk and interfacial properties exhibit a regular behavior when plotted as a function of $\varepsilon_{2} / \varepsilon_{1}$ and $\xi$. For all properties, a symmetry is found. It is shown in the supplementary material that this symmetry is likewise observed for the vapor pressure and vapor phase composition at constant temperature and liquid phase composition. The symmetry is also found in the matrix diagram of the density profiles in Fig. 6. This interesting regularity can be explained by a conformal solution theory, as shown in the following section.

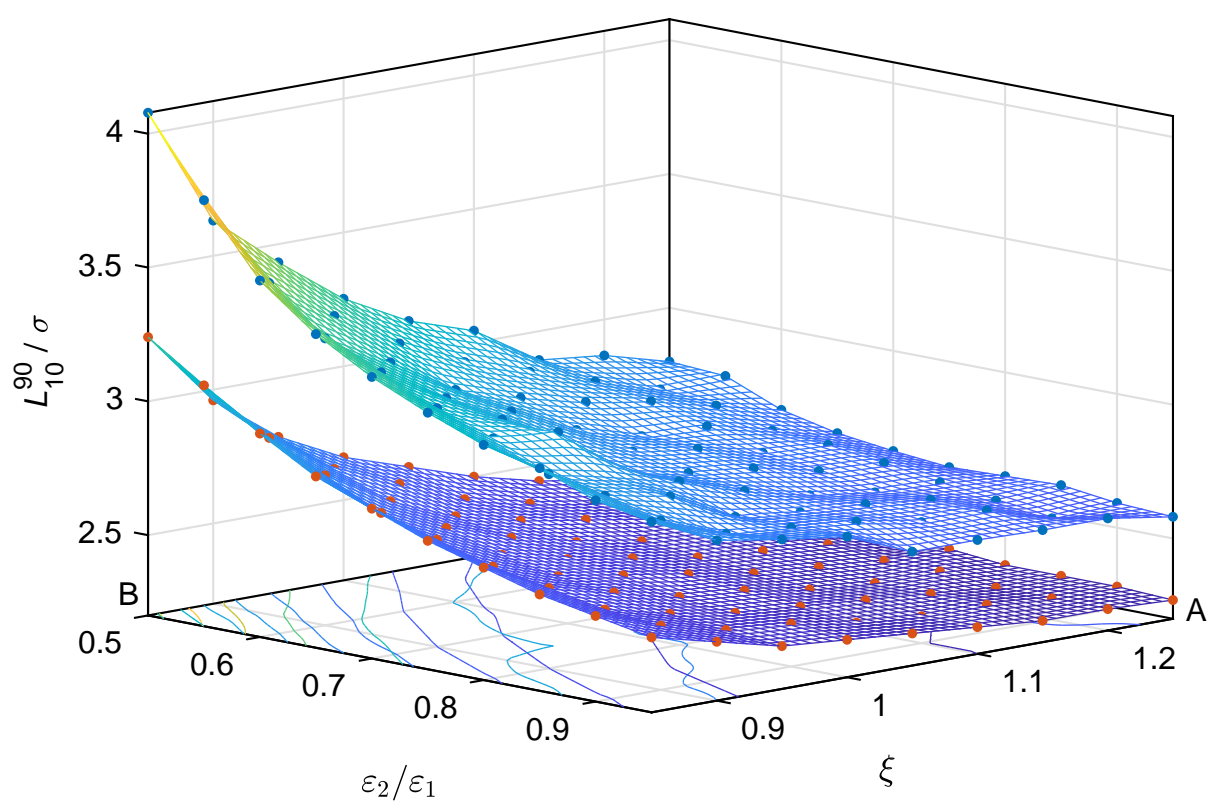

FIG. 10. Interfacial thickness $L_{10}^{90}$, as defined by Eq. (13), as a function of the binary interaction parameter $\xi$ and the ratio of the dispersion energies $\varepsilon_{2} / \varepsilon_{1}$ at $T=0.77 \varepsilon k_{\mathrm{B}}^{-1}$ and $x_{2}^{\prime}=0.05 \mathrm{~mol} \mathrm{~mol}^{-1}$. Results from MD: blue points; results from DGT: orange points. The surfaces are linear interpolations of the respective results. The color of the surfaces is coded by its height position. 


\section{CONFORMAL SOLUTION THEORY AT FLUID INTERFACES}

\section{A. Modeling the Regularity of VLE and Interfacial Properties}

The regularity of the properties of the studied binary LJTS mixtures (see previous section) can be explained with a conformal solution theory (CST) of the liquid phase. The regularity is thereby put into direct relation with the underlying molecular interactions. The employed CST is adopted from the literature and therefore only outlined here. For brevity, a mixture $n$ is defined by its coordinates in the $\xi, \varepsilon_{2} / \varepsilon_{1}$-plane as

$$
\underline{a}_{n}=\left(\xi_{n},\left(\varepsilon_{2} / \varepsilon_{1}\right)_{n}\right) .
$$

Our starting hypothesis is that the observed regularity is due to the mean dispersive interactions in the liquid phase. To validate this assumption, the configurational contribution of the internal energy $u_{\text {config }}^{\prime}$ of the saturated liquid phase is studied, where the total internal energy $u^{\prime}$ is the sum of the kinetic energy contribution $u_{\text {kin }}^{\prime}$ and the configurational contribution $u_{\text {config. }}^{\prime}$. For a pairwise additive intermolecular potential $v$, the configurational internal energy for a given point in the configurational space $\underline{r}$ is $u_{\text {config }}^{\prime}(\underline{r})=\sum_{k=1}^{N} \sum_{k>l}^{N} v\left(r_{k l}\right)$, where $N$ is the number of particles and $r_{k l}$ the distance between two particles $k$ and $l$. The configurational internal energy $u_{\text {config }}^{\prime}$ of a binary mixture can be calculated from rigorous statistical mechanics from the pair potential $v$ and the radial distribution function (RDF) $g(r)^{50,87,105}$

$$
u_{\text {config }}^{\prime}=\frac{U_{\text {config }}^{\prime}}{N}=\frac{1}{2} \rho^{\prime} \sum_{i=1}^{2} \sum_{j=1}^{2} x_{i}^{\prime} x_{j}^{\prime} \int_{0}^{\infty} g_{i j}(r) v_{i j}(r) r^{2} 4 \pi \mathrm{d} r,
$$

where $\rho^{\prime}$ is the total number density and $x_{i}^{\prime}$ the mole fraction of component $i, N$ the total number of particles, $g_{i j}$ the radial distribution function between two components $i$ and $j$, and $v_{i j}$ the pair potential energy. To compute and evaluate $u_{\text {config }}^{\prime}$ for a given mixture $\underline{a}_{n}$ at given $T=0.77 \varepsilon k_{\mathrm{B}}^{-1}$ and $x_{2}^{\prime}=0.05 \mathrm{~mol} \mathrm{~mol}^{-1}$, the conformal solution theory (CST) of Leland and co-workers ${ }^{52,53,106}$ is applied here, which goes back to the original work of van der Waals ${ }^{50,107}$ and Longuet-Higgins ${ }^{51}$. The CST of Leland and co-workers was developed to provide a statistical-mechanical framework to compute excess thermodynamic functions of mixing, from the intermolecular potentials and the radial distribution functions of the 
pure components ${ }^{51,52,108}$. The properties of the studied mixture are thereby approximated by those of a hypothetical pure fluid, indicated with the index 00 .

Using the assumptions of the conformal solution theory ${ }^{52,87}$ as summarized in the Appendix, Eq. (15) simplifies to:

$$
u_{\text {config }}^{\prime}=u_{\text {config }, 00}^{\prime}=\frac{1}{2} \rho^{\prime} \sum_{i=1}^{2} \sum_{j=1}^{2} x_{i}^{\prime} x_{j}^{\prime} \varepsilon_{i j} \sigma_{i j}^{3} \int_{0}^{\infty} g_{00}(\tilde{r}) v_{00}(\tilde{r}) \tilde{r}^{2} 4 \pi \mathrm{d} \tilde{r} .
$$

By identifying

$$
\sum_{i=1}^{2} \sum_{j=1}^{2} x_{i}^{\prime} x_{j}^{\prime} \varepsilon_{i j} \sigma_{i j}^{3}=\varepsilon_{00} \sigma_{00}^{3},
$$

Eq. (16) can then be written as

$$
u_{\text {config }, 00}^{\prime}=\varepsilon_{00} \sigma_{00}^{3} \cdot \frac{1}{2} \rho^{\prime} \int_{0}^{\infty} g_{00}(\tilde{r}) v_{00}(\tilde{r}) \tilde{r}^{2} 4 \pi \mathrm{d} \tilde{r} .
$$

Eq. (17) is the well-known first mixing rule of the van der Waals one-fluid theory, which is also used for modeling LJTS mixtures with the PeTS EOS ${ }^{5}$. Since $\sigma_{i j}=\sigma_{1}=1$ holds for the entire present study, a second mixing rule is obsolete here. The LJTS potential of the reference component 1 is used in Eq. (18), i.e. $v_{00}=v_{\mathrm{LJTS}}$.

As the dependency of $\rho^{\prime}$ on $\xi$ or $\varepsilon_{2} / \varepsilon_{1}$ can be neglected here in a good approximation, only the first term $\varepsilon_{00} \sigma_{00}^{3}$ in Eq. (18) depends on the mixture $\underline{a}_{n}$. The resulting configurational internal energy $u_{\text {config }}^{\prime}$ depends evidently directly on the chosen combination rule. In accordance with the MD, EOS, and DGT calculations, the modified Lorentz-Berthelot combination rule, cf. Eq. (3), was also employed for the CST.

A correlation for the radial distribution function of the Lennard-Jones fluid $g_{00}=g_{\mathrm{LJ}}$ was adopted from Morsali et al. ${ }^{109}$. They propose an empirical correlation of the Lennard-Jones $\mathrm{RDF}$ as a function of the temperature and the density $g_{\mathrm{LJ}}=g_{\mathrm{LJ}}(\tilde{r}, T, \rho)$. Morsali et al. ${ }^{109}$ developed an RDF correlation for the full Lennard-Jones fluid, whereas the present study investigates the Lennard-Jones truncated and shifted fluid. This is incorporated by employing a corresponding state principle, i.e. the Lennard-Jones RDF from Morsali et al. ${ }^{109}$ was calculated at the same reduced temperature and density with respect to its critical quantity, i.e. $T_{\text {red }}=T^{\mathrm{LJ}} / T_{\mathrm{c}}^{\mathrm{LJ}}=T^{\mathrm{LJTS}} / T_{\mathrm{c}}^{\mathrm{LJTS}}$ and $\rho_{\text {red }}=\rho^{\mathrm{LJ}} / \rho_{\mathrm{c}}^{\mathrm{LJ}}=\rho^{\mathrm{LJTS}} / \rho_{\mathrm{c}}^{\mathrm{LJTS}}$. The critical parameters were adopted from Vrabec et al. ${ }^{110}$ and Lotfi et al. ${ }^{66}$ for the LJTS and LJ fluid, respectively. The influence of the potential truncation on the RDF is therefore neglected. 
Morsali et al. ${ }^{109}$ compared results from their RDF with a large number of computer experiment data and found excellent agreement. A comparison of $u_{\text {config }}^{\prime}$ obtained from the PeTS EOS and the outlined CST is given in the supplementary material. They agree well; the absolute average deviation is $2 \%$.

In summary, Eq. (18) enables the computation of the configurational internal energy of binary LJTS mixtures $u_{\text {config }}^{\prime}(\underline{a})$ from CST, using only the RDF of the pure LJTS fluid $g_{\mathrm{LJTS}}(\tilde{r}, T, \rho)$ and the potential function $v_{\mathrm{LJTS}}(\tilde{r}) . u_{\text {config }}^{\prime}(\underline{a})$ is used here to characterize the mean interactions in the saturated liquid phase and subsequently put these in relation to the regularity of interfacial properties.

\section{B. Evaluation of the CST Model}

The configurational internal energy $u_{\text {config }}^{\prime}$ at $T=0.77 \varepsilon k_{\mathrm{B}}^{-1}$ and $x_{2}^{\prime}=0.05 \mathrm{~mol} \mathrm{~mol}^{-1}$, computed by Eq. (18), is shown in Fig. 11 as a function of $\xi$ and $\varepsilon_{2} / \varepsilon_{1}$ for all 90 investigated LJTS mixtures. Starting in corner $A$, the absolute value of the configurational internal energy $\left|u_{\text {config }}^{\prime}\right|$, and thereby also the magnitude of the mean dispersive interactions, steadily decreases with decreasing $\xi$ and decreasing $\varepsilon_{2} / \varepsilon_{1}$ towards corner $B$, which is a direct result of the molecular interactions. The internal energy of the liquid phase $u_{\text {config }}^{\prime}\left(\varepsilon_{2} / \varepsilon_{1}, \xi\right)$ obeys the same regular behavior as the bulk and interfacial properties, cf. Figs. 4, 5 and 7 - 10, i.e. radially shaped traces for $u_{\text {config }}^{\prime}=$ const. are found in the $\varepsilon_{2} / \varepsilon_{1}, \xi$-plane.

According to the discussion of Eq. (18), the configurational internal energy of a binary mixture $u_{\text {config }}^{\prime}$ at a given temperature and composition depends on the mixture type only via the term $\varepsilon_{00} \sigma_{00}^{3}$, that is defined by Eq. (17). Both the integral and the factor $\frac{1}{2} \rho^{\prime}$ in Eq. (18) can be merged to a constant $I$ at a given temperature and composition. Introducing $\zeta=\varepsilon_{2} / \varepsilon_{1}$, and employing the modified Berthelot combination rule, Eq. (18) can be written as

$$
\frac{u_{\text {config }}^{\prime}}{I}=\sum_{i=1}^{2} \sum_{j=1}^{2} x_{i}^{\prime} x_{j}^{\prime} \varepsilon_{i j} \sigma_{i j}^{3}=x_{1}^{\prime 2} \varepsilon_{1}+2 x_{1}^{\prime} x_{2}^{\prime} \varepsilon_{1} \xi \sqrt{\zeta}+{x_{2}^{\prime}}^{2} \varepsilon_{1} \zeta .
$$

The functional form of $u_{\text {config }}^{\prime}(\xi, \zeta)$ described by Eq. (19) yields the regularity with the approximatively radial traces of $u_{\text {config }}^{\prime}=$ const. in the $\varepsilon_{2} / \varepsilon_{1}, \xi$-plane, as depicted in Fig. 11 . Eq. (19) indicates that the regular traces that were observed in Figs. 4, 5 and $7-10$ are not strictly radial but obey a more complicated law. 


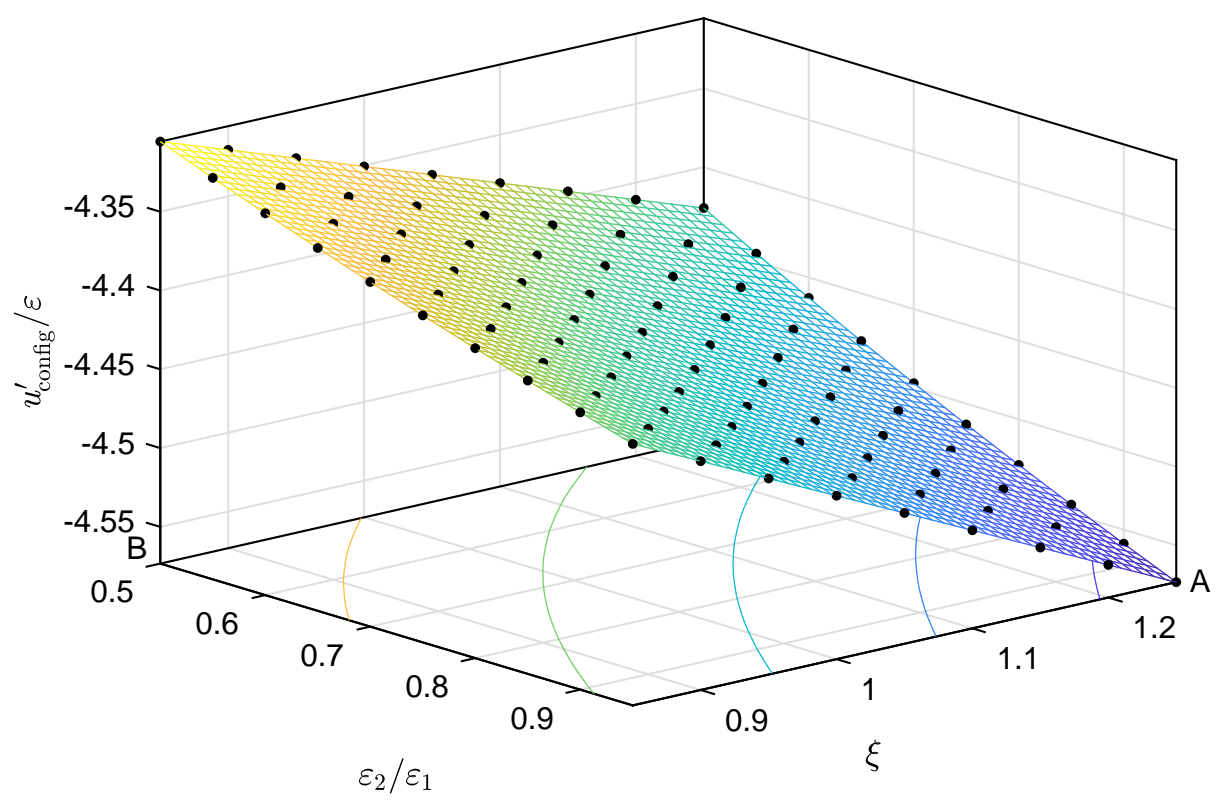

FIG. 11. Configurational internal energy of the saturated liquid phase $u_{\text {config }}^{\prime}$ calculated from the conformal solution theory Eq. (18) as a function of the binary interaction parameter $\xi$ and the ratio of the dispersion energies $\varepsilon_{2} / \varepsilon_{1}$ at $x_{2}^{\prime}=0.05 \mathrm{~mol} \mathrm{~mol}^{-1}$ and $T=0.77 \varepsilon k_{\mathrm{B}}^{-1}$. The surface is a linear interpolation of the data points. The color of the surface is coded by its height position.

In our case, $x_{1}^{\prime}, x_{2}^{\prime}$ and $\varepsilon_{1}$ are constant and the last term in Eq. (19) is small, as $x_{2}^{\prime}$ is small. Hence, the traces depicted in Fig. 11 as well as Figs. 4, 5 and 7 - 10 are basically curves in which $\xi \sqrt{\zeta}$ are constant. They only appear to be approximately radial in the plots used here, due to the investigated $\varepsilon_{2} / \varepsilon_{1}, \xi$ range.

The regular behavior found in $u_{\text {config }}^{\prime}\left(\varepsilon_{2} / \varepsilon_{1}, \xi\right)$ is the same regular behavior as obtained for the bulk and interfacial properties, cf. Figs. 4 - 10. Hence, the observed regularity (for both bulk and interfacial properties) is a consequence of the employed mixing and combination rule in $\mathrm{DGT}+\mathrm{EOS}$ and the combination rule in MD. However, the regularity and the shape of the traces evidently depend on the composition, cf. Eq. (19). The results from this work indicate that the van der Waals one-fluid theory mixing rule used in the PeTS EOS ${ }^{5,48}$ yields an excellent description of molecular simulation results for both bulk and interfacial mixture properties. The fact that the bulk and interfacial properties in the VLE are dominated by the liquid phase interactions and can be described by the CST can be exploited to find simple correlations of all studied properties. The hypothesis is here that they can be described as simple functions of the internal energy of the liquid phase. 
Figs. 12 and 13 show that this hypothesis is confirmed by the data from the present work: bulk and interfacial properties of the 90 LJTS mixtures are plotted as a function of

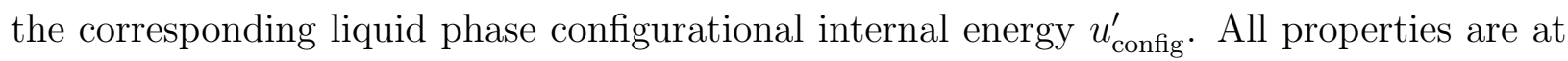
constant temperature and liquid phase composition. The results from both MD and DGT are shown. The investigated VLE bulk properties (Fig. 12) and interfacial properties (Fig. 13) from both MD and DGT are found to be a simple function of each mixture's liquid phase configurational internal energy, which strongly indicates that there is a direct link between them. For all studied properties, a simple relation is found, for both the results obtained from MD and those from EOS+DGT.

Instead of the internal energy from CST also the internal energy from the EOS or the force field could have been used for establishing plots like the ones shown in Figs. 12 and 13. The basic findings would have been the same, as the internal energies of the liquid phase determined by the three methods agree very well, see the supplementary material. However, using the CST gives further insight in the underlying mechanism, i.e. the fact that the choice of the combination rule is responsible for the regularity behavior, cf. Eq. (19).

A fit function $Y=Y\left(u_{\text {config }}^{\prime}\right)$ was parametrized to the DGT and EOS results for each bulk and interfacial property $Y=\left\langle H_{2,1}, p^{s}, x_{2}^{\prime \prime}, E_{2}, \Gamma_{2}^{(1)}, \gamma, L_{10}^{90}\right\rangle$. The mathematical forms and the parameters of the fitted functions are reported in the Appendix. The differences between the DGT and the MD results in Figs. 12 and 13 are due to differences in the data from these methods and not related to the CST, cf. Figs. 4, 5 and 7 - 10.

Fig. 12 shows the results for the Henry's law constant $H_{2,1}$, the vapor pressure $p^{s}$, and the gas phase mole fraction $x_{2}^{\prime \prime}$ for the conditions studied in the present work as a function of the corresponding liquid phase configurational internal energy. The three properties increase with decreasing mean dispersive interactions in the liquid phase, i.e. with decreasing absolute

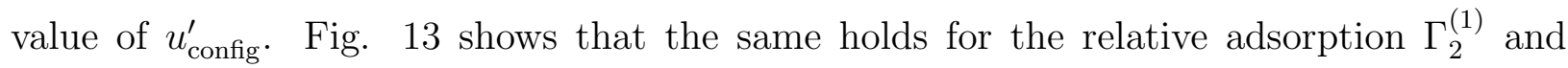
interfacial thickness $L_{10}^{90}$. The enrichment of the low-boiling component $E_{2}$ also generally increases with decreasing mean dispersive interactions, with the exception of the region near corner B. This exception is due to the difference in the number density, cf. Fig. 5. Only the surface tension $\gamma$ shows the inverse trend: it decreases with decreasing mean dispersive interactions. In all cases, simple functional relations between the studied properties and $u_{\text {config }}^{\prime}$ are found, which are however, of different types. The fitted functions for all seven investigated properties agree excellently with the underlying DGT results. Similar fits were 


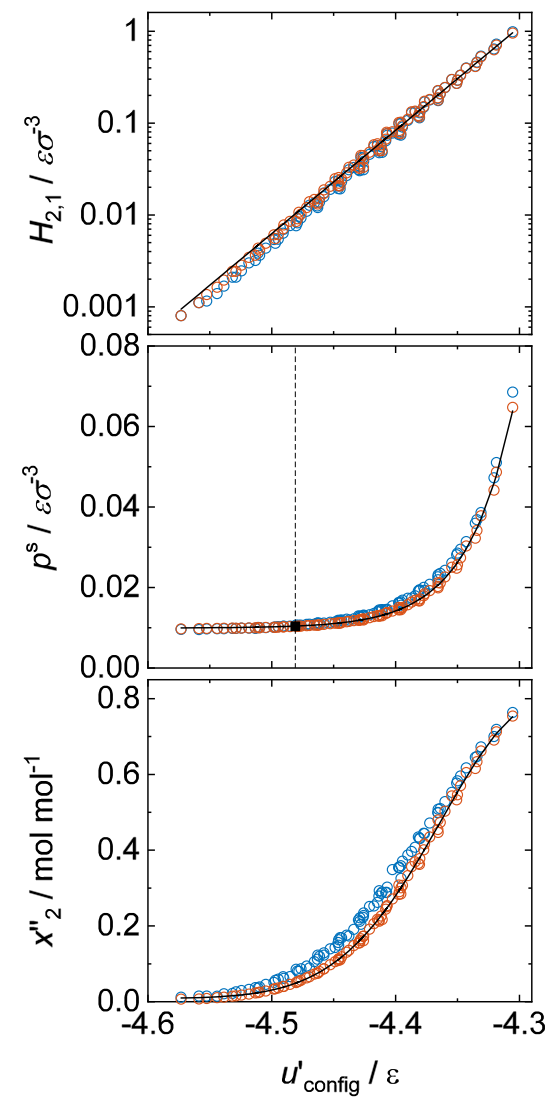

FIG. 12. Results for the conformal solution theory applied to the VLE properties (Henry's law constant $H_{2,1}$, vapor pressure $p^{s}$, and vapor phase composition $\left.x_{2}^{\prime \prime}\right)$ : The respective VLE property computed by MD (blue) and the EOS (orange) are shown as a function of the configurational internal energy of the saturated liquid phase $u_{\text {config }}^{\prime}$ as computed by Eq. (18). The black lines indicate the fitted functions $(\mathrm{C} 1)$ - (C3) (fitted to the DGT results). All results are at $x_{2}^{\prime}=$ $0.05 \mathrm{~mol} \mathrm{~mol}^{-1}$ and $T=0.77 \varepsilon k_{\mathrm{B}}^{-1}$. The dashed line indicates $u_{\text {config }}^{\prime}$ of the high-boiling pure component 1 computed by Eq. (18). The black symbol indicates the vapor pressure of the pure component 1 obtained from the EOS.

obtained for the MD results, but are not shown in Figs. 12 and 13 for clarity. The DGT and EOS results for all properties exhibit only low scattering around the respective fitted curves, i.e. the investigated bulk and interfacial properties are highly correlated with the liquid phase mean interactions. The scattering of the MD results is larger than that of the DGT and EOS results due to the fluctuations from the sampling during the simulation. The absolute average deviation (AAD) from the DGT and EOS results from the corresponding fitted curves were calculated for each property and are reported in Table II. 
The scattering of the DGT and EOS results for the vapor pressure $p^{s}$ and the vapor phase composition $x_{2}^{\prime \prime}$ around the respective fitted curve is fairly constant in the entire range of $u_{\text {config }}^{\prime}$, i.e. the range of investigated mixtures. In contrast to this, the scattering of the results for the interfacial properties around the respective fitted curves varies with varying $u_{\text {config }}^{\prime}$. The scattering is significantly lower for mixtures with large mean dispersive interactions than for those with low mean dispersive interactions, cf. results for $\Gamma_{2}^{(1)}, \gamma, L_{10}^{90}$ in Fig. 13. Furthermore, the scattering is lower for the surface tension and the interfacial thickness than for the relative adsorption and the enrichment, which indicates that the latter are less dominated by the liquid phase interactions but also influenced by the vapor phase interactions and the anisotropic interactions at the interface. This is supported by the corresponding AADs given in Table II.

High absolute values of $u_{\text {config }}^{\prime}$ are found for mixtures with strong mean liquid phase interactions. Eq. (19) shows that for the conditions that were studied in the present work, the variation of the mean liquid phase interaction stems mainly from the variation of the unlike dispersive interactions between component 1 and 2 . The higher the absolute value of $u_{\text {config }}^{\prime}$ is (left side in the diagrams of Figs. 12 and 13), the stronger is the attraction between the components 1 and 2. This explains why high absolute values of $u_{\text {config }}^{\prime}$ lead to low Henry's law constants $H_{2,1}$ (high solubilities of component 2 in component 1 ), low vapor pressures, and low concentrations of the component 2 in the gas phase, cf. Fig. 12. On the other hand, low absolute values of $u_{\text {config }}^{\prime}$ (weak 1 - 2 attraction) lead to high Henry's law constants $H_{2,1}$ (low solubilities of 2 in 1), high vapor pressures and high concentrations of the component 2 in the gas phase, cf. Fig. 12.

For mixtures with large mean dispersive interactions, a variation of $u_{\text {config }}^{\prime}$ (which goes in hand with a variation of $\varepsilon_{2} / \varepsilon_{1}$ and $\xi$ ) has only little influence on the bulk and interfacial properties. Note that $H_{2,1}$ is plotted on a ln-scale. Furthermore, the magnitude of the interfacial properties is small for these mixtures, e.g. small adsorption and enrichment of the low-boiling component.

Eq. (19) shows that the unlike dispersive interactions increase both with $\xi$ (which controls the dispersion energy $\varepsilon_{12}$ ) and with $\zeta=\varepsilon_{2} / \varepsilon_{1}$ (which controls the relative volatility). The unlike dispersive interactions are therefore high in corner $A$ and decrease going to corner $B$, cf. Figs. 4, 5 and 7 - 10. This also explains the observed phase behavior, cf. Fig. 3.

For high mean dispersive interactions (left side in the plot shown in Fig. 13), the in- 
terfacial thickness $L_{10}^{90}$ is low. For weaker interactions $L_{10}^{90}$ increases, as it might have been expected. Also the dependence of the surface tension $\gamma$ on the mean dispersive interactions is expected: it decreases with decreasing mean dispersive interactions.

Furthermore, Figs. 12 and 13 show that the developed CST also works in the limit of the pure component. The vapor pressure, surface tension and interfacial thickness of the pure component 1 computed from DGT+EOS at $T=0.77 \varepsilon k_{\mathrm{B}}^{-1}$ are shown as black sym-

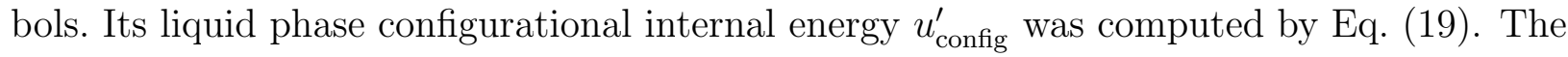
pure component values agree excellently with mixture values at the same $u_{\text {config. Hence, }}^{\prime}$ the vapor pressure, surface tension, and interfacial thickness of the pure component have the same values as those of mixtures with the same liquid phase mean interactions. Using these observables, a pure component could not be distinguished from these mixtures at the investigated temperature. Also, those mixtures have zero relative adsorption $\Gamma_{2}^{(1)}=0$, cf. Fig. 13. This strongly supports the picture of the one-fluid theory and the CST applied in this work.

Interestingly, the dependency of the interfacial properties on the liquid phase mean interactions is not symmetric, i.e. starting at the mixtures with $\Gamma_{2}^{(1)}=0$, an increase and decrease of $u_{\text {config }}^{\prime}$ has qualitatively different effects.

The surface tension that is reported here is the number for $x_{2}^{\prime}=0.05 \mathrm{~mol} \mathrm{~mol}^{-1}$. As the surface tension of the pure component 1 is the same in all cases, a decrease in the reported surface tension goes along with an increase of the gradient of the surface tension with varying $x_{2}^{\prime}$. It therefore follows from the Gibbs adsorption equation that the relative adsorption $\Gamma_{2}^{(1)}$ must increase, which is in line with the results shown in Fig. 13. High numbers for the relative adsorption of component 2 also go along with an enrichment of that component at the interface $E_{2}$, cf. Fig. 13 .

The increase of $\Gamma_{2}^{(1)}$ and $E_{2}$ with decreasing $u_{\text {config }}^{\prime}$ can also be understood based on Eq. (19). A decrease in $u_{\text {config }}^{\prime}$ stems either from a decrease in $\xi$ or from a decrease in $\varepsilon_{2} / \varepsilon_{1}$. The picture we invoke in the following is that of a dynamic equilibrium. Assume that a particle of component 2 is on the way of entering the liquid phase coming from the gas phase. The liquid phase contains basically only component 1 . The chance that the particle 2 will successfully enter the liquid phase decreases with decreasing mixed unlike dispersive interactions 1 - 2 in the liquid phase, i.e. with decreasing $\xi$ and $\varepsilon_{2} / \varepsilon_{1}$. Furthermore, low values of $\varepsilon_{2} / \varepsilon_{1}$ lead to high concentrations of component 2 in the gas phase (see Fig. 12), such that there are 
many particles of component 2 near the interface, which could in principle enter but face difficulties in doing so. This leads to the enrichment $E_{2}$ and the positive relative adsorption $\Gamma_{2}^{(1)}$. In a more colorful picture, the situation resembles that at the door of a disco: there are many people (particles 2) roaming outside and they are attracted by the disco's neon lights (the interface), but there are only few places available in the disco (in the liquid phase). Hence, there will be a crowd at the entrance (enrichment and adsorption). 


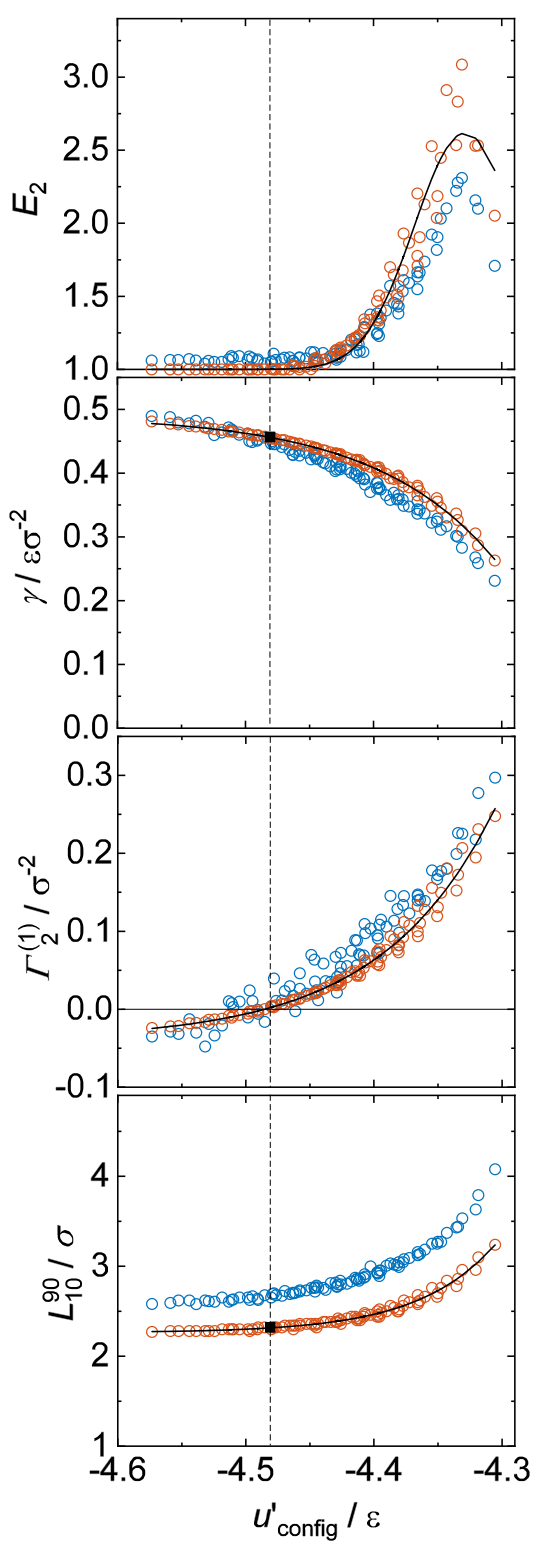

FIG. 13. Results for the conformal solution theory applied to the interfacial properties (enrichment $E_{2}$, relative adsorption $\Gamma_{2}^{(1)}$, surface tension $\gamma$, and interfacial thickness $\left.L_{10}^{90}\right)$ : The respective interfacial property computed by MD (blue) and DGT (orange) are shown as a function of the configurational internal energy of the saturated liquid phase $u_{\text {config }}^{\prime}$ as computed by Eq. (18). The black lines indicate the fitted functions (C1) - (C2) (fitted to the DGT results). All results are at $x_{2}^{\prime}=0.05 \mathrm{~mol} \mathrm{~mol}^{-1}$ and $T=0.77 \varepsilon k_{\mathrm{B}}^{-1}$. The dashed line indicates $u_{\text {config }}^{\prime}$ of the high-boiling pure component 1 computed by Eq. (18). Black symbols indicate the values for the pure component 1 obtained from the EOS+DGT. 


\section{CONCLUSIONS}

The vapor-liquid equilibrium and interfacial properties of binary Lennard-Jones mixtures (Henry's law constant, vapor pressure, composition, surface tension, adsorption, interfacial thickness, and enrichment) were determined for a broad range of mixtures of different types (zeotropic, low-boiling azeotrope, high-boiling azeotrope; subcritical, supercritical) by MD and EOS+DGT. The study was carried out at constant temperature and liquid phase composition. The results from MD and EOS+DGT were found to be in good agreement. The relation of both the VLE bulk properties and the interfacial properties with the underlying molecular interactions was investigated.

All considered bulk and interfacial properties exhibit a regular behavior when they are considered as a function of the variables $\varepsilon_{2} / \varepsilon_{1}$ and $\xi$, which describe the molecular interactions. A theory was developed which explains this behavior based on a conformal solution theory of the molecular interactions. It is based on the assumption that the behavior of the studied systems is dominated by the liquid phase mean interactions and that the liquid phase can be described with a one-fluid theory. The gas phase has only a minor influence. The basic finding is a monovariate relationship between the studied VLE properties and the mean liquid phase interactions, i.e. the configurational internal energy. This resembles the physical theory of the so-called entropy-scaling, which uses a monovariate relationship between transport coefficients and the entropy ${ }^{111}$. For the interfacial and bulk VLE properties, different combinations of $\varepsilon_{2} / \varepsilon_{1}$ and $\xi$ may lead to different types of phase behavior, but when the internal energy of the liquid phase is the same the value of the studied properties is the same. The theory also works in the limit of the pure component such that mixtures with the same liquid phase mean interactions as a pure component, have the same the vapor pressure, surface tension, and interfacial thickness as that pure component - and zero relative adsorption. The theory also enables predictions outside the range of the parameters $\varepsilon_{2} / \varepsilon_{1}$ and $\xi$ that was studied in the present work.

The interfacial enrichment, which is assumed to have an important influence on the mass transfer through interfaces, is found to be particularly high for Lennard-Jones mixtures that have low numbers for $\varepsilon_{2} / \varepsilon_{1}$ and for $\xi$. Furthermore, the enrichment exhibits a maximum

for mixtures with $\Delta \rho_{2}=\rho_{2}^{\prime}-\rho_{2}^{\prime \prime}=0$, which forces all adsorption at the interface into an enrichment. Both large enrichment and large relative adsorption of the low-boiling compo- 
nent is found for mixtures with relatively low mean interactions in the liquid phase, which also goes in hand with low solubilities, i.e. wide-boiling mixtures that tend to form lowboiling azeotropes. Such mixtures also tend to show liquid-liquid phase splits. Systems with liquid-liquid equilibria were, however, not investigated here as this would have overloaded the study. It would be interesting to extend the study to such systems in future work.

As only data for constant temperature and liquid phase composition were taken in the present work, it would be interesting to extend the study to other temperatures and compositions and test if the monovariate relationship established in this work also holds for those dimensions.

\section{ACKNOWLEDGMENTS}

The authors gratefully acknowledge funding of the present work by the ERC Advanced Grant ENRICO (grant No. 694807). The simulations were carried out on the ELWE supercomputer at Regional University Computing Center Kaiserslautern (RHRK) under the grant TUKL-TLMV. The present research was conducted under the auspices of the BoltzmannZuse Society of Computational Molecular Engineering (BZS).

\section{Appendix A: Supplementary Information}

Supplementary data can be found at [online DOI-link $]^{112}$. The numerical values for the bulk properties from MD and the PeTS EOS are listed there as well as the Henry's law constant data. Also details on the MD simulation procedures are given.

\section{Appendix B: Assumptions made in the Conformal Solution Theory}

The main assumptions of the employed conformal solution theory, cf. Eqs. (15) - (18), are summarized in the following. In Eq. (15) $4 \pi \rho^{\prime} g(r) \mathrm{d} r=n^{\prime}(r) \mathrm{d} r$ can be identified ${ }^{105}$ as the mean number of particles $n^{\prime}$ in a range between $r$ and $r+\mathrm{d} r$. The configurational internal energy $u_{\text {config }}^{\prime}$ is obtained by integrating $n^{\prime}(r) \cdot v(r)$ over all $r$ ranges and weighted by the respective mole fraction; the factor $1 / 2$ ensures that each pair interaction is only counted once. In general the three radial distribution functions $g_{11}, g_{12}$, and $g_{22}$ are a functions of the type of both components $(\varepsilon, \sigma)$, the composition, the temperature, the density, and the 
distance $r$ between two particles ${ }^{50,113-115}$, i.e. $g_{i j}=g_{i j}\left(\varepsilon_{i j}, \sigma_{i j}, \underline{x}, T, \rho, r\right)$. It is known that the RDF is only weakly depending on the composition and the dispersion energies of the components $^{113,114}$.

For the one-fluid CST, the properties of a mixture are approximated by those of a hypothetical pure fluid. The molecular interactions of that hypothetical fluid are to be conformal to those of the pure components that constitute the mixture, i.e. their pair potentials $v_{i j}$ must be described ${ }^{87}$ by the same mathematical function $v_{i j}=\varepsilon_{i j} v\left(r / \sigma_{i j}\right)$. The hypothetical pure fluid - in our case a spherical Lennard-Jones pair potential - is then characterized by the two interaction parameters $\sigma_{00}$ and $\varepsilon_{00}$ and a single radial distribution function $g_{00}=g_{00}(\tilde{r}, T, \rho)^{50,52}$. The central assumption in the 'one-fluid' CST is that the radial distribution functions $g_{i j}(r)$ scale with the respective size parameter $\sigma_{i j}$, i.e.

$$
g_{i j}\left(r / \sigma_{i j}\right)=g_{00}\left(\tilde{r} / \sigma_{00}\right) \text {, }
$$

which includes a coordinate transformation ${ }^{87} r \rightarrow \tilde{r}$. Thereby, the internal energy of the liquid phase of a binary mixture can be written as Eq. (16).

\section{Appendix C: Fitted Functions for Bulk and Interfacial Properties in CST}

For each of the seven considered properties $Y=\left\langle H_{2,1}, p^{s}, x_{2}^{\prime \prime}, E_{2}, \Gamma_{2}^{(1)}, \gamma, L_{10}^{90}\right\rangle$, a simple correlation function was parameterized to describe $Y=Y\left(u_{\text {config }}^{\prime}\right)$. The DGT results were used for this fit since they do not exhibit stochastic scattering as the MD results do. Three function types were used:

$$
\begin{aligned}
& Y /[Y]=\hat{Y}+a_{1} \cdot \exp \left(\frac{u_{\text {config }}^{\prime} / \varepsilon-a_{2}}{a_{3}}\right), \\
& Y /[Y]=\hat{Y}+a_{1} \cdot \exp \left(-0.5\left(\frac{u_{\text {config }}^{\prime} / \varepsilon-a_{2}}{a_{3}}\right)^{2}\right), \\
& Y /[Y]=\hat{Y} \cdot \exp \left(a_{3} u_{\text {config }}^{\prime} / \varepsilon\right),
\end{aligned}
$$

where $\hat{Y}$ and $a_{i}$ are fitting parameters. $[Y]$ indicates the corresponding units of $Y$ as $[Y]=$ $\left\langle\varepsilon \sigma^{-3}, \varepsilon \sigma^{-3}, \mathrm{~mol} \mathrm{~mol}^{-1}, 1, \sigma^{-2}, \varepsilon \sigma^{-2}, \sigma\right\rangle$. The obtained numeric values for $\hat{Y}$ and $a_{i}$ are summarized in Table II. Eq. (C1) was used for correlating $p^{s}, \Gamma_{2}^{(1)}, \gamma, L_{10}^{90}$, Eq. (C2) for $x_{2}^{\prime \prime}, E_{2}$, and Eq. (C3) for correlating $H_{2,1}$. The absolute average deviations (AAD) between the fitted curves and the DGT results are also given in Table II. 
TABLE I: Interfacial properties of the investigated binary LJTS mixtures. Results from MD and DGT+PeTS EOS. The temperature is $T=0.77 \varepsilon k_{\mathrm{B}}^{-1}$. The DGT results are calculated at the liquid phase composition $x_{2}^{\prime}=0.05 \mathrm{~mol} \mathrm{~mol}^{-1}$. The exact MD liquid phase composition is given in the $2^{\text {nd }}$ column. The other columns are from left to right: the surface tension, the relative adsorption, the enrichment of the lowboiling component 2 , and the interfacial thickness. The number in the parentheses indicates the statistical uncertainty in the last decimal digit.

\begin{tabular}{|c|c|c|c|c|c|c|c|c|c|}
\hline \multirow[t]{2}{*}{$\varepsilon_{2} / \varepsilon_{1}$} & \multirow[t]{2}{*}{$x_{2}^{\prime} / \mathrm{mol} \mathrm{mol}^{-1}$} & \multicolumn{2}{|c|}{$\gamma / \varepsilon \sigma^{-2}$} & \multicolumn{2}{|c|}{$\Gamma_{2}^{(1)} / \sigma^{-2}$} & \multicolumn{2}{|c|}{$E_{2}$} & \multicolumn{2}{|c|}{$L_{10}^{90} / \sigma$} \\
\hline & & $\mathrm{MD}$ & EOS & $\mathrm{MD}$ & EOS & $\mathrm{MD}$ & EOS & $\mathrm{MD}$ & EOS \\
\hline \multicolumn{10}{|c|}{$\xi=0.85$} \\
\hline 0.5 & $0.058(2)$ & $0.23(3)$ & 0.263 & $0.30(3)$ & 0.248 & $1.71(3)$ & 2.05 & $4.08(9)$ & 3.24 \\
\hline 0.55 & $0.058(1)$ & $0.26(3)$ & 0.287 & $0.28(2)$ & 0.231 & $2.10(3)$ & 2.53 & $3.8(1)$ & 3.10 \\
\hline 0.6 & $0.063(3)$ & $0.28(2)$ & 0.310 & $0.23(4)$ & 0.207 & $2.3(2)$ & 3.09 & $3.53(9)$ & 2.96 \\
\hline 0.65 & $0.062(2)$ & $0.32(2)$ & 0.330 & $0.18(4)$ & 0.181 & $2.1(1)$ & 2.91 & $3.37(8)$ & 2.84 \\
\hline 0.7 & $0.061(1)$ & $0.32(2)$ & 0.349 & $0.18(2)$ & 0.156 & $1.92(8)$ & 2.53 & $3.3(1)$ & 2.76 \\
\hline 0.75 & $0.063(2)$ & $0.34(2)$ & 0.365 & $0.15(3)$ & 0.133 & $1.66(8)$ & 2.20 & $3.16(7)$ & 2.68 \\
\hline 0.8 & $0.062(2)$ & $0.35(2)$ & 0.379 & $0.15(3)$ & 0.112 & $1.6(1)$ & 1.93 & $3.08(8)$ & 2.62 \\
\hline 0.85 & $0.061(1)$ & $0.36(1)$ & 0.392 & $0.15(2)$ & 0.094 & $1.57(7)$ & 1.70 & $3.03(8)$ & 2.56 \\
\hline 0.9 & $0.065(2)$ & $0.38(2)$ & 0.403 & $0.11(4)$ & 0.077 & $1.3(1)$ & 1.51 & $2.95(4)$ & 2.52 \\
\hline 0.95 & $0.064(1)$ & $0.39(2)$ & 0.413 & $0.11(4)$ & 0.063 & $1.29(9)$ & 1.34 & $2.91(5)$ & 2.48 \\
\hline \multicolumn{10}{|c|}{$\xi=0.9$} \\
\hline 0.5 & $0.059(4)$ & $0.27(2)$ & 0.305 & $0.22(3)$ & 0.195 & $2.16(5)$ & 2.53 & $3.63(9)$ & 2.96 \\
\hline 0.55 & $0.059(3)$ & $0.30(1)$ & 0.326 & $0.23(2)$ & 0.172 & $2.3(1)$ & 2.83 & $3.44(7)$ & 2.86 \\
\hline 0.6 & $0.059(1)$ & $0.31(2)$ & 0.346 & $0.18(2)$ & 0.149 & $2.0(1)$ & 2.45 & $3.27(9)$ & 2.76 \\
\hline 0.65 & $0.062(1)$ & $0.34(2)$ & 0.363 & $0.14(3)$ & 0.128 & $1.74(7)$ & 2.13 & $3.19(7)$ & 2.66 \\
\hline 0.7 & $0.061(2)$ & $0.35(2)$ & 0.378 & $0.12(2)$ & 0.108 & $1.6(1)$ & 1.87 & $3.09(6)$ & 2.60 \\
\hline
\end{tabular}




\begin{tabular}{|c|c|c|c|c|c|c|c|c|c|}
\hline \multirow[t]{2}{*}{$\varepsilon_{2} / \varepsilon_{1}$} & \multirow[t]{2}{*}{$x_{2}^{\prime} / \mathrm{mol} \mathrm{mol}^{-1}$} & \multicolumn{2}{|c|}{$\gamma / \varepsilon \sigma^{-2}$} & \multicolumn{2}{|c|}{$\Gamma_{2}^{(1)} / \sigma^{-2}$} & \multicolumn{2}{|c|}{$E_{2}$} & \multicolumn{2}{|c|}{$L_{10}^{90} / \sigma$} \\
\hline & & $\mathrm{MD}$ & DGT & MD & DGT & $\mathrm{MD}$ & DGT & MD & DGT \\
\hline 0.75 & $0.062(2)$ & $0.37(2)$ & 0.391 & $0.11(3)$ & 0.090 & $1.46(8)$ & 1.65 & $3.02(6)$ & 2.54 \\
\hline 0.8 & $0.062(1)$ & $0.38(2)$ & 0.402 & $0.09(2)$ & 0.074 & $1.38(6)$ & 1.47 & $2.93(6)$ & 2.50 \\
\hline 0.85 & $0.063(2)$ & $0.38(3)$ & 0.413 & $0.10(1)$ & 0.060 & $1.3(1)$ & 1.32 & $2.89(4)$ & 2.46 \\
\hline 0.9 & $0.065(2)$ & $0.40(2)$ & 0.422 & $0.07(3)$ & 0.048 & $1.20(7)$ & 1.19 & $2.83(5)$ & 2.44 \\
\hline 0.95 & $0.066(1)$ & $0.42(2)$ & 0.429 & $0.03(3)$ & 0.037 & $1.09(6)$ & 1.09 & $2.80(4)$ & 2.42 \\
\hline \multicolumn{10}{|c|}{$\xi=0.95$} \\
\hline 0.5 & $0.057(1)$ & $0.30(2)$ & 0.337 & $0.20(3)$ & 0.152 & $2.22(5)$ & 2.53 & $3.43(7)$ & 2.78 \\
\hline 0.55 & $0.059(1)$ & $0.33(2)$ & 0.355 & $0.17(2)$ & 0.131 & $1.90(7)$ & 2.19 & $3.27(8)$ & 2.70 \\
\hline 0.6 & $0.061(3)$ & $0.34(2)$ & 0.372 & $0.14(2)$ & 0.111 & $1.7(1)$ & 1.91 & $3.14(9)$ & 2.62 \\
\hline 0.65 & $0.061(1)$ & $0.36(3)$ & 0.386 & $0.13(1)$ & 0.092 & $1.53(8)$ & 1.68 & $3.05(8)$ & 2.56 \\
\hline 0.7 & $0.062(2)$ & $0.37(3)$ & 0.399 & $0.11(3)$ & 0.076 & $1.4(1)$ & 1.49 & $3.01(8)$ & 2.50 \\
\hline 0.75 & $0.061(1)$ & $0.37(2)$ & 0.410 & $0.10(2)$ & 0.062 & $1.38(3)$ & 1.34 & $3.00(5)$ & 2.46 \\
\hline 0.8 & $0.064(2)$ & $0.40(2)$ & 0.419 & $0.09(2)$ & 0.049 & $1.21(8)$ & 1.22 & $2.87(8)$ & 2.44 \\
\hline 0.85 & $0.065(1)$ & $0.41(2)$ & 0.428 & $0.05(3)$ & 0.038 & $1.11(8)$ & 1.12 & $2.84(7)$ & 2.40 \\
\hline 0.9 & $0.067(2)$ & $0.42(2)$ & 0.435 & $0.04(3)$ & 0.028 & $1.07(6)$ & 1.04 & $2.74(6)$ & 2.38 \\
\hline 0.95 & $0.067(1)$ & $0.42(2)$ & 0.442 & $0.07(2)$ & 0.019 & $1.12(4)$ & 1.00 & $2.76(7)$ & 2.36 \\
\hline \multicolumn{10}{|l|}{$\xi=1$} \\
\hline 0.5 & $0.059(2)$ & $0.32(2)$ & 0.361 & $0.17(3)$ & 0.119 & $1.82(8)$ & 2.04 & $3.25(9)$ & 2.66 \\
\hline 0.55 & $0.062(1)$ & $0.34(2)$ & 0.377 & $0.14(3)$ & 0.100 & $1.55(8)$ & 1.78 & $3.13(7)$ & 2.60 \\
\hline 0.6 & $0.062(1)$ & $0.36(1)$ & 0.392 & $0.10(2)$ & 0.082 & $1.43(7)$ & 1.57 & $2.99(7)$ & 2.52 \\
\hline 0.65 & $0.062(2)$ & $0.37(2)$ & 0.404 & $0.11(2)$ & 0.067 & $1.35(1)$ & 1.40 & $2.95(6)$ & 2.48 \\
\hline 0.7 & $0.065(1)$ & $0.39(3)$ & 0.415 & $0.07(4)$ & 0.053 & $1.12(7)$ & 1.27 & $2.87(6)$ & 2.44 \\
\hline 0.75 & $0.065(2)$ & $0.41(2)$ & 0.424 & $0.06(2)$ & 0.041 & $1.13(5)$ & 1.16 & $2.81(5)$ & 2.42 \\
\hline 0.8 & $0.066(2)$ & $0.41(2)$ & 0.433 & $0.04(3)$ & 0.031 & $1.09(6)$ & 1.08 & $2.81(6)$ & 2.38 \\
\hline 0.85 & $0.067(2)$ & $0.43(3)$ & 0.440 & $0.02(5)$ & 0.022 & $1.05(7)$ & 1.02 & $2.72(7)$ & 2.36 \\
\hline 0.9 & $0.068(2)$ & $0.43(2)$ & 0.446 & $0.05(3)$ & 0.013 & $1.08(6)$ & 1.00 & $2.72(8)$ & 2.36 \\
\hline 0.95 & $0.068(2)$ & $0.45(2)$ & 0.452 & $0.02(3)$ & 0.006 & $1.08(7)$ & 1.00 & $2.73(4)$ & 2.34 \\
\hline
\end{tabular}

Continued on next page 


\begin{tabular}{|c|c|c|c|c|c|c|c|c|c|}
\hline \multirow[t]{2}{*}{$\varepsilon_{2} / \varepsilon_{1}$} & \multirow[t]{2}{*}{$x_{2}^{\prime} / \mathrm{mol} \mathrm{mol}^{-1}$} & \multicolumn{2}{|c|}{$\gamma / \varepsilon \sigma^{-2}$} & \multicolumn{2}{|c|}{$\Gamma_{2}^{(1)} / \sigma^{-2}$} & \multicolumn{2}{|c|}{$E_{2}$} & \multicolumn{2}{|c|}{$L_{10}^{90} / \sigma$} \\
\hline & & $\mathrm{MD}$ & DGT & $\mathrm{MD}$ & DGT & $\mathrm{MD}$ & DGT & MD & DGT \\
\hline \multicolumn{10}{|c|}{$\xi=1.05$} \\
\hline 0.5 & $0.059(2)$ & $0.36(2)$ & 0.380 & $0.15(3)$ & 0.094 & $1.6(1)$ & 1.71 & $3.13(6)$ & 2.58 \\
\hline 0.55 & $0.061(1)$ & $0.36(2)$ & 0.395 & $0.13(3)$ & 0.076 & $1.44(7)$ & 1.51 & $3.01(8)$ & 2.50 \\
\hline 0.6 & $0.063(1)$ & $0.38(2)$ & 0.407 & $0.09(2)$ & 0.061 & $1.25(6)$ & 1.36 & $2.92(7)$ & 2.46 \\
\hline 0.65 & $0.064(1)$ & $0.40(2)$ & 0.418 & $0.07(2)$ & 0.048 & $1.17(6)$ & 1.23 & $2.84(6)$ & 2.42 \\
\hline 0.7 & $0.065(2)$ & $0.41(2)$ & 0.428 & $0.07(3)$ & 0.036 & $1.13(6)$ & 1.14 & $2.80(4)$ & 2.40 \\
\hline 0.75 & $0.067(2)$ & $0.42(3)$ & 0.436 & $0.06(2)$ & 0.026 & $1.11(7)$ & 1.07 & $2.76(9)$ & 2.38 \\
\hline 0.8 & $0.067(1)$ & $0.43(2)$ & 0.443 & $0.02(2)$ & 0.017 & $1.08(5)$ & 1.02 & $2.73(6)$ & 2.36 \\
\hline 0.85 & $0.067(1)$ & $0.44(2)$ & 0.449 & $0.03(1)$ & 0.010 & $1.08(3)$ & 1.00 & $2.69(4)$ & 2.34 \\
\hline 0.9 & $0.068(2)$ & $0.45(2)$ & 0.455 & $0.04(4)$ & 0.003 & $1.11(9)$ & 1.00 & $2.70(9)$ & 2.32 \\
\hline 0.95 & $0.069(1)$ & $0.45(2)$ & 0.460 & $-0.01(2)$ & -0.003 & $1.07(4)$ & 1.00 & $2.63(5)$ & 2.32 \\
\hline \multicolumn{10}{|l|}{$\xi=1.1$} \\
\hline 0.5 & $0.061(1)$ & $0.37(2)$ & 0.396 & $0.08(3)$ & 0.073 & $1.38(7)$ & 1.49 & $3.05(5)$ & 2.50 \\
\hline 0.55 & $0.063(2)$ & $0.39(2)$ & 0.409 & $0.07(2)$ & 0.058 & $1.24(9)$ & 1.34 & $2.93(7)$ & 2.44 \\
\hline 0.6 & $0.065(2)$ & $0.41(2)$ & 0.420 & $0.06(3)$ & 0.044 & $1.12(7)$ & 1.22 & $2.86(6)$ & 2.42 \\
\hline 0.65 & $0.065(2)$ & $0.41(2)$ & 0.430 & $0.04(3)$ & 0.033 & $1.08(5)$ & 1.13 & $2.77(8)$ & 2.38 \\
\hline 0.7 & $0.066(1)$ & $0.42(3)$ & 0.438 & $0.01(3)$ & 0.023 & $1.04(5)$ & 1.06 & $2.77(4)$ & 2.36 \\
\hline 0.75 & $0.067(2)$ & $0.43(2)$ & 0.445 & $0.03(3)$ & 0.014 & $1.08(4)$ & 1.02 & $2.73(6)$ & 2.34 \\
\hline 0.8 & $0.068(1)$ & $0.44(2)$ & 0.452 & $0.01(2)$ & 0.007 & $1.07(4)$ & 1.00 & $2.67(5)$ & 2.32 \\
\hline 0.85 & $0.069(1)$ & $0.46(2)$ & 0.457 & $-0.02(4)$ & 0.000 & $1.04(6)$ & 1.00 & $2.68(6)$ & 2.32 \\
\hline 0.9 & $0.070(1)$ & $0.46(2)$ & 0.462 & $-0.01(3)$ & -0.006 & $1.07(3)$ & 1.00 & $2.65(5)$ & 2.30 \\
\hline 0.95 & $0.070(2)$ & $0.47(2)$ & 0.466 & $0.01(3)$ & -0.011 & $1.09(4)$ & 1.00 & $2.63(7)$ & 2.30 \\
\hline \multicolumn{10}{|c|}{$\xi=1.15$} \\
\hline 0.5 & $0.063(2)$ & $0.38(3)$ & 0.409 & $0.07(2)$ & 0.057 & $1.2(1)$ & 1.33 & $2.89(3)$ & 2.44 \\
\hline 0.55 & $0.063(1)$ & $0.40(2)$ & 0.421 & $0.09(3)$ & 0.043 & $1.17(6)$ & 1.22 & $2.85(7)$ & 2.40 \\
\hline 0.6 & $0.065(1)$ & $0.40(2)$ & 0.431 & $0.07(2)$ & 0.031 & $1.11(4)$ & 1.13 & $2.82(7)$ & 2.38 \\
\hline 0.65 & $0.066(1)$ & $0.43(2)$ & 0.439 & $0.04(2)$ & 0.021 & $1.08(5)$ & 1.06 & $2.77(4)$ & 2.36 \\
\hline
\end{tabular}

Continued on next page 


\begin{tabular}{|c|c|c|c|c|c|c|c|c|c|}
\hline \multirow[t]{2}{*}{$\varepsilon_{2} / \varepsilon_{1}$} & \multirow[t]{2}{*}{$x_{2}^{\prime} / \mathrm{mol} \mathrm{mol}^{-1}$} & \multicolumn{2}{|c|}{$\gamma / \varepsilon \sigma^{-2}$} & \multicolumn{2}{|c|}{$\Gamma_{2}^{(1)} / \sigma^{-2}$} & \multicolumn{2}{|c|}{$E_{2}$} & \multicolumn{2}{|c|}{$L_{10}^{90} / \sigma$} \\
\hline & & $\mathrm{MD}$ & DGT & $\mathrm{MD}$ & DGT & $\mathrm{MD}$ & DGT & MD & DGT \\
\hline 0.7 & $0.068(2)$ & $0.43(2)$ & 0.447 & $0.00(5)$ & 0.013 & $1.04(5)$ & 1.02 & $2.71(7)$ & 2.34 \\
\hline 0.75 & $0.068(1)$ & $0.45(3)$ & 0.453 & $0.01(3)$ & 0.005 & $1.06(5)$ & 1.00 & $2.68(5)$ & 2.32 \\
\hline 0.8 & $0.069(1)$ & $0.45(2)$ & 0.459 & $0.00(3)$ & -0.001 & $1.06(3)$ & 1.00 & $2.64(7)$ & 2.30 \\
\hline 0.85 & $0.070(2)$ & $0.46(1)$ & 0.464 & $0.01(3)$ & -0.007 & $1.09(6)$ & 1.00 & $2.65(8)$ & 2.30 \\
\hline 0.9 & $0.070(1)$ & $0.46(2)$ & 0.468 & $-0.02(2)$ & -0.012 & $1.05(5)$ & 1.00 & $2.65(7)$ & 2.30 \\
\hline 0.95 & $0.071(1)$ & $0.47(2)$ & 0.472 & $-0.05(2)$ & -0.017 & $1.04(4)$ & 1.00 & $2.62(5)$ & 2.28 \\
\hline \multicolumn{10}{|l|}{$\xi=1.2$} \\
\hline 0.5 & $0.064(2)$ & $0.39(2)$ & 0.420 & $0.07(3)$ & 0.043 & $1.13(8)$ & 1.23 & $2.87(9)$ & 2.40 \\
\hline 0.55 & $0.067(2)$ & $0.42(2)$ & 0.431 & $0.02(3)$ & 0.031 & $1.07(6)$ & 1.13 & $2.78(5)$ & 2.38 \\
\hline 0.6 & $0.066(2)$ & $0.43(2)$ & 0.440 & $0.06(2)$ & 0.021 & $1.12(5)$ & 1.07 & $2.76(6)$ & 2.34 \\
\hline 0.65 & $0.067(1)$ & $0.43(2)$ & 0.447 & $0.01(2)$ & 0.012 & $1.06(3)$ & 1.02 & $2.71(4)$ & 2.34 \\
\hline 0.7 & $0.069(1)$ & $0.45(2)$ & 0.454 & $0.00(2)$ & 0.004 & $1.05(3)$ & 1.00 & $2.69(5)$ & 2.32 \\
\hline 0.75 & $0.069(1)$ & $0.45(2)$ & 0.460 & $0.01(2)$ & -0.002 & $1.08(3)$ & 1.00 & $2.67(4)$ & 2.30 \\
\hline 0.8 & $0.070(1)$ & $0.47(3)$ & 0.465 & $0.00(3)$ & -0.008 & $1.08(4)$ & 1.00 & $2.63(5)$ & 2.30 \\
\hline 0.85 & $0.070(2)$ & $0.46(3)$ & 0.469 & $-0.03(2)$ & -0.013 & $1.05(4)$ & 1.00 & $2.61(5)$ & 2.28 \\
\hline 0.9 & $0.070(1)$ & $0.48(3)$ & 0.473 & $-0.03(1)$ & -0.018 & $1.06(3)$ & 1.00 & $2.58(7)$ & 2.28 \\
\hline 0.95 & $0.070(1)$ & $0.48(2)$ & 0.476 & $-0.03(3)$ & -0.022 & $1.06(3)$ & 1.00 & $2.62(7)$ & 2.28 \\
\hline \multicolumn{10}{|c|}{$\xi=1.25$} \\
\hline 0.5 & $0.065(1)$ & $0.41(2)$ & 0.430 & $0.05(3)$ & 0.032 & $1.09(4)$ & 1.15 & $2.80(5)$ & 2.36 \\
\hline 0.55 & $0.067(1)$ & $0.42(2)$ & 0.439 & $0.02(2)$ & 0.021 & $1.06(3)$ & 1.08 & $2.79(8)$ & 2.32 \\
\hline 0.6 & $0.067(1)$ & $0.43(2)$ & 0.447 & $0.02(1)$ & 0.012 & $1.06(2)$ & 1.03 & $2.70(4)$ & 2.30 \\
\hline 0.65 & $0.069(1)$ & $0.45(2)$ & 0.454 & $0.00(3)$ & 0.004 & $1.05(3)$ & 1.00 & $2.65(7)$ & 2.30 \\
\hline 0.7 & $0.069(1)$ & $0.45(2)$ & 0.460 & $0.02(2)$ & -0.003 & $1.09(4)$ & 1.00 & $2.63(4)$ & 2.28 \\
\hline 0.75 & $0.070(1)$ & $0.47(2)$ & 0.465 & $0.01(3)$ & -0.008 & $1.07(3)$ & 1.00 & $2.61(8)$ & 2.30 \\
\hline 0.8 & $0.070(1)$ & $0.48(2)$ & 0.470 & $-0.02(1)$ & -0.014 & $1.06(2)$ & 1.00 & $2.61(6)$ & 2.28 \\
\hline 0.85 & $0.071(1)$ & $0.48(2)$ & 0.474 & $-0.01(2)$ & -0.018 & $1.07(4)$ & 1.00 & $2.62(7)$ & 2.28 \\
\hline 0.9 & $0.070(1)$ & $0.49(2)$ & 0.477 & $-0.03(2)$ & -0.022 & $1.07(3)$ & 1.00 & $2.59(6)$ & 2.28 \\
\hline
\end{tabular}

Continued on next page 


\begin{tabular}{cccccccccc}
\hline \hline$\varepsilon_{2} / \varepsilon_{1}$ & $x_{2}^{\prime} / \mathrm{mol} \mathrm{mol}^{-1}$ & \multicolumn{2}{c}{$\gamma / \varepsilon \sigma^{-2}$} & \multicolumn{2}{c}{$\Gamma_{2}^{(1)} / \sigma^{-2}$} & \multicolumn{2}{c}{$E_{2}$} & \multicolumn{2}{c}{$L_{10}^{90} / \sigma$} \\
& & $\mathrm{MD}$ & $\mathrm{DGT}$ & $\mathrm{MD}$ & $\mathrm{DGT}$ & $\mathrm{MD}$ & $\mathrm{DGT}$ & $\mathrm{MD}$ & $\mathrm{DGT}$ \\
\hline 0.95 & $0.071(1)$ & $0.49(2)$ & 0.481 & $-0.04(1)$ & -0.024 & $1.06(4)$ & 1.00 & $2.58(8)$ & 2.28 \\
\hline \hline
\end{tabular}


TABLE II. Parameters for the correlations (C1) - (C3).

\begin{tabular}{llllll}
\hline \hline & $\hat{Y}$ & $a_{1}$ & $a_{2}$ & $a_{3}$ & $\mathrm{AAD}$ \\
\hline${ }^{(\mathrm{c})} H_{2,1}$ & $2.32 \mathrm{E}+48$ & - & 25.87506 & $9.5 \%$ \\
${ }^{(\mathrm{a})} p^{s}$ & 0.00993 & $1.05 \mathrm{E}+49$ & - & -0.03718 & $1.8 \%$ \\
${ }^{(\mathrm{b})} x_{2}^{\prime \prime}$ & 0.00823 & 0.77992 & -4.28011 & 0.08256 & $3.5 \%$ \\
${ }^{(\mathrm{b})} E_{2}$ & 1 & 1.61673 & -4.32865 & 0.03954 & $3.9 \%$ \\
${ }^{(\mathrm{a})} \Gamma_{2}^{(1)}$ & -0.03928 & $3.19 \mathrm{E}+20$ & - & -0.08891 & $12.8 \%$ \\
${ }^{(\mathrm{a})} \gamma$ & 0.49078 & $-2.03 \mathrm{E}+19-$ & -0.09371 & $0.6 \%$ \\
${ }^{(\mathrm{a})} L_{10}^{90}$ & 2.26152 & $8.42 \mathrm{E}+30$ & - & -0.06044 & $0.7 \%$ \\
\hline \hline
\end{tabular}

(a) uses Eq. (C1)

(b) uses Eq. (C2)

(c) uses Eq. (C3) 


\section{REFERENCES}

${ }^{1}$ S. Becker, S. Werth, M. Horsch, K. Langenbach, and H. Hasse, "Interfacial tension and adsorption in the binary system ethanol and carbon dioxide: Experiments, molecular simulation and density gradient theory," Fluid Phase Equilibria 427, 476 (2016).

2 "Dortmund data bank (accessed june 2019)," 2019, www.ddbst.com.

${ }^{3}$ J. S. Rowlinson and B. Widom, Molecular Theory of Capillarity (Dover Publications, New York, 1982).

${ }^{4}$ R. Evans, Fundamentals of Inhomogeneous Fluids, edited by D. Henderson (Marcel Dekker, New York, 1992).

${ }^{5}$ S. Stephan, K. Langenbach, and H. Hasse, "Interfacial properties of binary LennardJones mixtures by molecular simulations and density gradient theory," The Journal of Chemical Physics 150, 174704 (2019).

${ }^{6}$ C. Klink and J. Gross, "A density functional theory for vapor-liquid interfaces of mixtures using the perturbed-chain polar statistical associating fluid theory equation of state," Industrial \& Engineering Chemistry Research 53, 6169 (2014).

${ }^{7}$ R. Nagl, P. Zimmermann, and T. Zeiner, "Interfacial mass transfer in water-toluene systems," Journal of Chemical \& Engineering Data 0, null (0).

${ }^{8}$ S. Stephan, K. Langenbach, and H. Hasse, "Enrichment of components at vapour-liquid interfaces: A study by molecular simulation and density gradient theory," Chemical Engineering Transactions 69, 295-300 (2018).

${ }^{9}$ F. J. Martinez-Ruiz and F. J. Blas, "Interfacial properties of binary mixtures of squarewell molecules from Monte Carlo simulation," The Journal of Chemical Physics 144, 154705 (2016).

${ }^{10}$ F. J. Blas and L. F. Vega, "Thermodynamic behaviour of homonuclear and heteronuclear Lennard-Jones chains with association sites from simulation and theory," Molecular Physics 92, 135 (1997).

${ }^{11}$ D. J. Lee, M. M. Telo da Gama, and K. E. Gubbins, "The vapour-liquid interface for a Lennard-Jones model of argon-krypton mixtures," Molecular Physics 53, 1113-1130 (1984).

${ }^{12}$ D. J. Lee, M. M. Telo da Gama, and K. E. Gubbins, "Adsorption and surface tension reduction at the vapor-liquid interface," The Journal of Physical Chemistry 89, 1514-1519 
(1985).

${ }^{13}$ A. Mejía, J. C. Pàmies, D. Duque, H. Segura, and L. F. Vega, "Phase and interface behaviors in type-I and type-V Lennard-Jones mixtures: Theory and simulations," The Journal of Chemical Physics 123, 034505 (2005).

${ }^{14}$ J. C. Neyt, A. Wender, V. Lachet, A. Ghoufi, and P. Malfreyt, "Molecular modeling of the liquid-vapor interfaces of a multi-component mixture: Prediction of the coexisting densities and surface tensions at different pressures and gas compositions," The Journal of Chemical Physics 139, 024701 (2013).

${ }^{15}$ S. Eckelsbach and J. Vrabec, "Fluid phase interface properties of acetone, oxygen, nitrogen and their binary mixtures by molecular simulation," Physical Chemistry Chemical Physics 17, 27195-27203 (2015).

${ }^{16}$ M. M. Telo da Gama and R. Evans, "Theory of the liquid-vapour interface of a binary mixture of Lennard-Jones fluids," Molecular Physics 41, 1091-1112 (1980).

${ }^{17}$ M. M. Telo da Gama and R. Evans, "Surface segregation and surface tension at the liquidvapour interface of a binary mixture of Lennard-Jones fluids," Faraday Symposia of the Chemical Society 16, 45-58 (1981).

${ }^{18}$ S. P. Protsenko, V. G. Baidakov, and V. M. Bryukhanov, "Binary Lennard-Jones mixtures with highly asymmetric interactions of the components. 2. effect of the particle size on phase equilibria and properties of liquid-gas interfaces," Fluid Phase Equilibr. 430, 67-74 (2016).

${ }^{19}$ S. P. Protsenko and V. G. Baidakov, "Binary Lennard-Jones mixtures with highly asymmetric interactions of the components. 1. effect of the energy parameters on phase equilibria and properties of liquid-gas interfaces," Fluid Phase Equilibr. 429, 242-253 (2016).

${ }^{20}$ V. G. Baidakov and S. P. Protsenko, "Molecular-dynamics investigation of phase equilibrium and surface tension in argon-neon system," J. Phys. Chem. C 112, 17231-17234 (2008).

${ }^{21}$ V. G. Baidakov, A. M. Kaverin, and M. N. Khotienkova, "Surface tension of ethanemethane solutions: 1. experiment and thermodynamic analysis of the results," Fluid Phase Equilibr. 356, 90-95 (2013).

${ }^{22}$ V. G. Baidakov and M. N. Khotienkova, "Surface tension of methane-nitrogen solutions: 2. description in the framework of the van der Waals gradient theory," Fluid Phase Equilibria 425, 402-410 (2016). 
${ }^{23}$ F. Llovell, A. Galindo, F. J. Blas, and G. Jackson, "Classical density functional theory for the prediction of the surface tension and interfacial properties of fluids mixtures of chain molecules based on the statistical associating fluid theory for potentials of variable range," The Journal of Chemical Physics 133, 024704 (2010).

${ }^{24}$ C. I. Poser and I. C. Sanchez, "Interfacial tension theory of low and high molecular weight liquid mixtures," Macromolecules 14, 361-370 (1981).

${ }^{25}$ O. G. Niño-Amézquita, S. Enders, P. T. Jaeger, and R. Eggers, "Interfacial properties of mixtures containing supercritical gases," The Journal of Supercritical Fluids 55, 724 (2010).

${ }^{26}$ O. G. Niño-Amézquita, D. van Putten, and S. Enders, "Phase equilibrium and interfacial properties of water $+\mathrm{CO}_{2}$ mixtures," Fluid Phase Equilibria 332, 40 (2012).

${ }^{27}$ O. G. Niño-Amézquita, S. Enders, P. T. Jaeger, and R. Eggers, "Measurement and prediction of interfacial tension of binary mixtures," Industrial \& Engineering Chemistry Research 49, 592-601 (2010).

${ }^{28}$ O. G. Niño-Amézquita and S. Enders, "Phase equilibrium and interfacial properties of water+methane mixtures," Fluid Phase Equilibria 407, 143-151 (2016).

${ }^{29}$ F. Biscay, A. Ghoufi, V. Lachet, and P. Malfreyt, "Monte Carlo simulations of the pressure dependence of the water-acid gas interfacial tensions," The Journal of Physical Chemistry B 113, 14277-14290 (2009).

${ }^{30} \mathrm{~S}$. Stephan and H. Hasse, "Influence of dispersive long range interactions on properties of vapour-liquid interfaces of binary Lennard-Jones mixtures," Molecular Physics (in press) (2019).

${ }^{31}$ M. Sahimi and B. N. Taylor, "Surface tension of binary liquid-vapor mixtures: A comparison of mean-field and scaling theories," The Journal of Chemical Physics 95, 6749-6761 (1991).

${ }^{32}$ X.-S. Li, J.-M. Liu, and D. Fu, "Investigation of interfacial tensions for carbon dioxide aqueous solutions by perturbed-chain statistical associating fluid theory combined with density-gradient theory," Industrial \& Engineering Chemistry Research 47, 8911-8917 (2008).

${ }^{33}$ T. Lafitte, B. Mendiboure, M. M. Pieiro, D. Bessires, and C. Miqueu, "Interfacial properties of water $/ \mathrm{CO}_{2}$ : A comprehensive description through a gradient theory SAFT-VR Mie approach," The Journal of Physical Chemistry B 114, 11110-11116 (2010). 
${ }^{34}$ O. Lobanova, A. Mejía, G. Jackson, and E. A. Müller, "SAFT-i force field for the simulation of molecular fluids 6: Binary and ternary mixtures comprising water, carbon dioxide, and n-alkanes," The Journal of Chemical Thermodynamics 93, 320-336 (2016).

${ }^{35}$ H. Lin, Y.-Y. Duan, and Q. Min, "Gradient theory modeling of surface tension for pure fluids and binary mixtures," Fluid Phase Equilibria 254, 75 (2007).

${ }^{36}$ E. A. Müller and A. Mejía, "Interfacial properties of selected binary mixtures containing n-alkanes," Fluid Phase Equilibria 282, 68-81 (2009).

${ }^{37}$ C. Cumicheo, M. Cartes, H. Segura, E. A. Müller, and A. Mejía, "High-pressure densities and interfacial tensions of binary systems containing carbon dioxide + n-alkanes: (ndodecane, n-tridecane, n-tetradecane)," Fluid Phase Equilibria 380, 82 (2014).

${ }^{38}$ L. M. Pereira, A. Chapoy, R. Burgass, and B. Tohidi, "Measurement and modelling of high pressure density and interfacial tension of (gas + n-alkane) binary mixtures," The Journal of Chemical Thermodynamics 97, 55-69 (2016).

${ }^{39}$ W. A. Fouad and L. F. Vega, "The phase and interfacial properties of azeotropic refrigerants: the prediction of aneotropes from molecular theory," Physical Chemistry Chemical Physics 19, 8977-8988 (2017).

${ }^{40}$ J. M. Garrido, H. Quinteros-Lama, M. M. Pieiro, A. Mejía, and H. Segura, "On the phase and interface behavior along the three-phase line of ternary Lennard-Jones mixtures: A collaborative approach based on square gradient theory and molecular dynamics simulations," The Journal of Chemical Physics 141, 014503 (2014).

${ }^{41}$ W. Li and Z. Jin, "Molecular dynamics simulation of natural gas-water interfacial tensions over wide range of pressures," Fuel 236, 480-492 (2019).

${ }^{42}$ C. Miqueu, J. M. Miguez, M. M. Pineiro, T. Lafitte, and B. Mendiboure, "Simultaneous application of the gradient theory and Monte Carlo molecular simulation for the investigation of methane/water interfacial properties," The Journal of Physical Chemistry B 115, 9618-9625 (2011).

${ }^{43}$ V. Mazur, L. Boshkov, and V. Murakhovsky, "Global phase behaviour of binary mixtures of Lennard-Jones molecules," Physics Letters A 104, 415-418 (1984).

${ }^{44}$ P. H. van Konynenburg and R. L. Scott, "Critical lines and phase equilibria in binary van der Waals mixtures," Philosophical Transactions of the Royal Society of London A 298, 495-540 (1980). 
${ }^{45}$ R. Privat and J.-N. Jaubert, "Classification of global fluid-phase equilibrium behaviors in binary systems," Chemical Engineering Research and Design 91, 1807-1839 (2013).

${ }^{46}$ U. K. Deiters and I. L. Pegg, "Systematic investigation of the phase behavior in binary fluid mixtures. I. calculations based on the Redlich-Kwong equation of state," The Journal of Chemical Physics 90, 6632-6641 (1989).

${ }^{47}$ T. Kraska and U. K. Deiters, "Systematic investigation of the phase behavior in binary fluid mixtures. II. calculations based on the Carnahan-Starling-Redlich-Kwong equation of state," The Journal of Chemical Physics 96, 539-547 (1992).

${ }^{48}$ M. Heier, S. Stephan, J. Liu, W. G. Chapman, H. Hasse, and K. Langenbach, "Equation of state for the Lennard-Jones truncated and shifted fluid with a cut-off radius of $2.5 \sigma$ based on perturbation theory and its applications to interfacial thermodynamics," Molecular Physics 116, 2083-2094 (2018).

${ }^{49}$ D. Berthelot, "Sur le mélange des gaz," Comptes rendus hebdomadaires des séances de l'Académie des Sciences 126, 1703-1706 (1898).

${ }^{50} \mathrm{G}$. Mansoori, "Radial distribution functions and their role in modeling of mixtures behavior," Fluid Phase Equilibria 87, 1-22 (1993).

${ }^{51}$ H. Longuet-Higgins, "The statistical thermodynamics of multicomponent systems," Proceedings of the Royal Society of London A 205, 247-269 (1951).

${ }^{52}$ T. W. Leland, J. S. Rowlinson, and G. A. Sather, "Statistical thermodynamics of mixtures of molecules of different sizes," Transactions of the Faraday Society 64, 1447-1460 (1968).

${ }^{53}$ T. W. Leland, J. S. Rowlinson, G. A. Sather, and I. D. Watson, "Statistical thermodynamics of two-fluid models of mixtures," Transactions of the Faraday Society 65, 20342043 (1969).

${ }^{54}$ G. A. Mansoori and T. W. Leland, "Statistical thermodynamics of mixtures. a new version for the theory of conformal solution," Journal of the Chemical Society, Faraday Transactions 2: 68, 320-344 (1972).

${ }^{55}$ K. C. Mo, K. E. Gubbins, G. Jacucci, and I. R. McDonald, "The radial distribution function in fluid mixtures: Conformal solution theory and molecular dynamics results," Molecular Physics 27, 1173-1183 (1974).

${ }^{56}$ K. C. Mo and K. E. Gubbins, "Conformal solution theory for viscosity and thermal conductivity of mixtures," Molecular Physics 31, 825-847 (1976). 
${ }^{57}$ G. Rutkai, M. Thol, R. Span, and J. Vrabec, "How well does the Lennard-Jones potential represent the thermodynamic properties of noble gases?" Mol. Phys. 115, 1104-1121 (2017).

${ }^{58}$ S. Stephan, M. Horsch, J. Vrabec, and H. Hasse, "MolMod - an open access database of force fields for molecular simulations of fluids," Mol. Simulat. 45, 806-814 (2019).

${ }^{59}$ M. Horsch, H. Hasse, A. K. Shchekin, A. Agarwal, S. Eckelsbach, J. Vrabec, E. A. Müller, and G. Jackson, "Excess equimolar radius of liquid drops," Physical Review E 85, 031605 (2012).

${ }^{60}$ S. Becker, H. M. Urbassek, M. Horsch, and H. Hasse, "Contact angle of sessile drops in Lennard-Jones systems," Langmuir 30, 13606 (2014).

${ }^{61}$ M. Horsch, S. Miroshnichenko, and J. Vrabec, "Steady-state molecular dynamics simulation of vapour to liquid nucleation with McDonald's daemon," Journal of Physical Studies 13, 4004 (2009).

${ }^{62}$ M. Thol, G. Rutkai, R. Span, J. Vrabec, and R. Lustig, "Equation of state for the Lennard-Jones truncated and shifted model fluid," International Journal of Thermophysics 36, 25 (2015).

${ }^{63}$ S. Stephan, J. Liu, K. Langenbach, W. G. Chapman, and H. Hasse, "Vapor-liquid interface of the Lennard-Jones truncated and shifted fluid: Comparison of molecular simulation, density gradient theory, and density functional theory," J. Phys. Chem. C 122, 24705-24715 (2018).

${ }^{64}$ M. P. Allen and D. J. Tildesley, Computer Simulation of Liquids (Oxford University Press, Oxford, 1989).

${ }^{65}$ S. Stephan, M. Thol, J. Vrabec, and H. Hasse, "Thermophysical properties of the lennardjones fluid: Database and data assessment," Journal of Chemical Information and Modeling 59, 4248-4265 (2019).

${ }^{66}$ A. Lotfi, J. Vrabec, and J. Fischer, "Vapour liquid equilibria of the Lennard-Jones fluid from the NpT plus test particle method," Mol. Phys. 76, 1319-1333 (1992).

${ }^{67}$ A. Trokhymchuk and J. Alejandre, "Computer simulations of liquid/vapor interface in Lennard-Jones fluids: Some questions and answers," J. Chem. Phys. 111, 8510-8523 (1999).

${ }^{68}$ B. Smit, "Phase diagrams of Lennard-Jones fluids," J. Chem. Phys. 96, 8639-8640 (1992). 
${ }^{69}$ D. O. Dunikov, S. P. Malyshenko, and V. V. Zhakhovskii, "Corresponding states law and molecular dynamics simulations of the Lennard-Jones fluid," J. Chem. Phys. 115, 6623-6631 (2001).

${ }^{70}$ S. Werth, S. V. Lishchuk, M. Horsch, and H. Hasse, "The influence of the liquid slab thickness on the planar vapor-liquid interfacial tension," Physica A 392, 2359 (2013).

${ }^{71}$ M. Guo, D.-Y. Peng, and B. C.-Y.Lu, "On the long-range corrections to computer simulation results for the Lennard-Jones vapor-liquid interface," Fluid Phase Equilibr. 130, 19-30 (1997).

${ }^{72}$ M. J. P. Nijmeijer, A. F. Bakker, C. Bruin, and J. H. Sikkenk, "A molecular dynamics simulation of the Lennard-Jones liquid-vapor interface," J. Chem. Phys. 89, 3789-3792 (1988).

${ }^{73}$ V. Baidakov, G. Chernykh, and S. Protsenko, "Effect of the cut-off radius of the intermolecular potential on phase equilibrium and surface tension in Lennard-Jones systems," Chem. Phys. Lett. 321, 315-320 (2000).

${ }^{74}$ R. Evans, J. R. Henderson, D. C. Hoyle, A. O. Parry, and Z. A. Sabeur, "Asymptotic decay of liquid structure: Oscillatory liquid-vapour density profiles and the Fisher-Widom line," Molecular Physics 80, 755-775 (1993).

${ }^{75}$ R. Evans, "Oscillatory behaviour of density profiles: Relevance for fluid interfacial phenomena," Berichte der Bunsengesellschaft für physikalische Chemie 98, 345-352 (1994).

${ }^{76}$ H. A. Lorentz, "Ueber die Anwendung des Satzes vom Virial in der kinetischen Theorie der Gase," Annalen Der Physik 248, 127-136 (1881).

${ }^{77}$ T. Schnabel, J. Vrabec, and H. Hasse, "Unlike Lennard-Jones parameters for vapor-liquid equilibria," Journal of Molecular Liquids 135, 170-178 (2007).

${ }^{78} \mathrm{~J}$. Vrabec, Y. lin Huang, and H. Hasse, "Molecular models for 267 binary mixtures validated by vapor-liquid equilibria: A systematic approach," Fluid Phase Equilibria 279, 120-135 (2009).

${ }^{79}$ C. Niethammer, S. Becker, M. Bernreuther, M. Buchholz, W. Eckhardt, A. Heinecke, S. Werth, H.-J. Bungartz, C. W. Glass, H. Hasse, J. Vrabec, and M. Horsch, "ls1 mardyn: The massively parallel molecular dynamics code for large systems," Journal of Chemical Theory Computation 10, 4455 (2014).

${ }^{80}$ D. Fincham, "Leapfrog rotational algorithms," Molecular Simulation 8, 165-178 (1992). 
${ }^{81}$ J. Walton, D. J. Tildesley, J. S. Rowlinson, and J. R. Henderson, "The pressure tensor at the planar surface of a liquid," Molecular Physics 48, 1357-1368 (1983).

${ }^{82}$ J. G. Kirkwood and F. P. Buff, "The statistical mechanical theory of surface tension," The Journal of Chemical Physics 17, 338-343 (1949).

${ }^{83}$ B. Widom, "Some topics in the theory of fluids," J. Chem. Phys. 39, 2808 (1963).

${ }^{84}$ G. Rutkai, A. Köster, G. Guevara-Carrion, T. Janzen, M. Schappals, C. W. Glass, M. Bernreuther, A. Wafai, S. Stephan, M. Kohns, S. Reiser, S. Deublein, M. Horsch, H. Hasse, and J. Vrabec, "ms2: A molecular simulation tool for thermodynamic properties, release 3.0," Comput. Phys. Commun. 221, 343-351 (2017).

${ }^{85}$ K. S. Shing, K. E. Gubbins, and K. Lucas, "Henry constants in non-ideal fluid mixtures," Molecular Physics 65, 1235-1252 (1988).

${ }^{86} \mathrm{~J}$. A. Barker and D. Henderson, "Perturbation theory and equation of state for fluids. II. a successful theory of liquids," The Journal of Chemical Physics 47, 4714 (1967).

${ }^{87}$ J.-P. Hansen and I. McDonald, Theory of Simple Liquids, 4th ed. (Academic Press, 2013).

${ }^{88}$ J. Gross and G. Sadowski, "Application of perturbation theory to a hard-chain reference fluid: an equation of state for square-well chains," Fluid Phase Equilibria 168, 183 (2000).

${ }^{89}$ S. K. Shibata and S. I. Sandler, "Critical evaluation of equation of state mixing rules for the prediction of high-pressure phase equilibria," Industrial \& Engineering Chemistry Research 28, 1893-1898 (1989).

${ }^{90}$ C. Miqueu, B. Mendiboure, A. Graciaa, and J. Lachaise, "Modelling of the surface tension of pure components with the gradient theory of fluid interfaces: a simple and accurate expression for the influence parameters," Fluid Phase Equilibria 207, 225 (2003).

${ }^{91}$ P. M. Cornelisse, C. J. Peters, and J. de Swaan Arons, "On the fundamentals of the gradient theory of van der Waals," The Journal of Chemical Physics 106, 9820-9834 (1997).

${ }^{92}$ P. M. Cornelisse, The Square Gradient Theory Applied Simultaneous Modelling of Interfacial Tension and Phase Behaviour, Ph.D. thesis, Technische Universiteit Delft (1997).

${ }^{93}$ B. S. Carey, L. E. Scriven, and H. T. Davis, "Semiempirical theory of surface tension of binary systems," AIChE Journal 26, 705-711 (1980).

${ }^{94}$ C. Miqueu, B. Mendiboure, C. Graciaa, and J. Lachaise, "Modelling of the surface tension of binary and ternary mixtures with the gradient theory of fluid interfaces," Fluid Phase Equilibria 218, 189-203 (2004). 
${ }^{95}$ X. Mu, F. Frank, F. O. Alpak, and W. G. Chapman, "Stabilized density gradient theory algorithm for modeling interfacial properties of pure and mixed systems," Fluid Phase Equilibria 435, 118-130 (2017).

${ }^{96}$ X. Liang, M. L. Michelsen, and G. M. Kontogeorgis, "Pitfalls of using the geometricmean combining rule in the density gradient theory," Fluid Phase Equilibria 415, 75-83 (2016).

${ }^{97}$ J. W. Gibbs, The Scientific Papers of J. W. Gibbs (Dover Publications, 1961).

${ }^{98}$ M. M. Telo da Gama and R. Evans, "The structure and surface tension of the liquidvapour interface near the upper critical end point of a binary mixture of Lennard-Jones fluids," Molecular Physics 48, 229-250 (1983).

${ }^{99}$ T. Wadewitz and J. Winkelmann, "Density functional theory: Structure and interfacial properties of binary mixtures," Berichte der Bunsengesellschaft für physikalische Chemie 100, 1825-1832 (1996).

${ }^{100}$ J. Lekner and J. R. Henderson, "Surface tension and energy of a classical liquid-vapour interface," Molecular Physics 34, 333-359 (1977).

${ }^{101}$ I. A. McLure, B. Edmonds, and M. Lal, "Extremes in surface tension of fluorocarbon + hydrocarbon mixtures," Nature Physical Science 241, 71-71 (1973).

${ }^{102}$ I. A. McLure, V. A. M. Soares, and A. M. Williamson, "Total surface segregation. a fresh look at the Gibbs adsorption isotherm for binary liquid mixtures." Langmuir 9, 2190-2201 (1993).

${ }^{103}$ M. J. Osborne and D. J. Lacks, "Surface segregation in liquid mixtures with strong interspecies attraction," Physical Review E 70, 010501 (2004).

${ }^{104}$ R. Defay, I. Prigogine, A. Bellmans, and D. H. Everett, Surface Tension and Adsorption (Longmans, London, 1966).

${ }^{105}$ T. Hill, Statistical Mechanics (McGraw-Hill Book, 1956).

${ }^{106}$ G. D. Fisher and T. W. Leland, "Corresponding states principle using shape factors," Industrial \& Engineering Chemistry Fundamentals 9, 537-544 (1970).

${ }^{107}$ J. D. van der Waals, Over de Continuiteit van Den Gas En Vloeistoftoestand. (On the Continuity of the Gas and Liquid State), Ph.D. thesis, Leiden University (1873).

${ }^{108}$ J. S. Rowlinson and F. L. Swinton, Liquids and Liquid Mixtures (Butterworth, London, 1982). 
${ }^{109}$ A. Morsali, E. Goharshadi, G. Ali Mansoori, and M. Abbaspour, "An accurate expression for radial distribution function of the Lennard-Jones fluid," Chemical Physics 310, 11-15 (2005).

${ }^{110}$ J. Vrabec, G. K. Kedia, G. Fuchs, and H. Hasse, "Comprehensive study of the vapourliquid coexistence of the truncated and shifted Lennard-Jones fluid including planar and spherical interface properties," Molecular Physics 104, 1509 (2006).

${ }^{111}$ Y. Rosenfeld, "Relation between the transport coefficients and the internal entropy of simple systems," Phys. Rev. A 15, 2545-2549 (1977).

112 "See supplemental material at [url will be inserted by publisher] for numeric values of the data presented in this work.".

${ }^{113}$ E. Matteoli and G. A. Mansoori, "A simple expression for radial distribution functions of pure fluids and mixtures," The Journal of Chemical Physics 103, 4672-4677 (1995).

${ }^{114}$ L. L. Lee and H. M. Hulburt, "Correlation functions of classical fluids. i. the radial distribution functions of mixtures of Kihara molecules in the Percus-Yevick approximation and their thermodynamic functions," The Journal of Chemical Physics 58, 44-60 (1973).

${ }^{115}$ M. L. Huber and J. F. Ely, "Properties of Lennard-Jones mixtures at various temperatures and energy ratios with a size ratio of two," technical note 1331 (NIST, 1989). 